Chinese Journal of Organic Chemistry

ARTICLE

\title{
新型香豆素类衍生物的合成及抗肿瘤活性研究
}

\author{
时 蕾 ${ }^{a}$ 李子秋 ${ }^{a}$ 崔金金金 ${ }^{b}$ 朱 挺 ${ }^{b}$ 庞晓静 ${ }^{b}$ 李龙辉 ${ }^{a}$ \\ 罗德福 ${ }^{a}$ 刘方芳 ${ }^{b}$ 赵冰玉 ${ }^{b}$ 龙 跃*, $a$ 张赛扬*,c \\ ( ${ }^{a}$ 郑州大学化学学院 郑州 450001) \\ $\left({ }^{b}\right.$ 郑州大学药学院 郑州 450001) \\ ( ${ }^{c}$ 郑州大学基础医学院 郑州 450001)
}

\begin{abstract}
摘要 为了寻找结构新颖、活性较好的抗肿瘤化合物, 设计合成了 19 个未见文献报道的 3,4,5-三甲氧基苯基香豆素类 化合物，并用核磁共振(NMR)和高分辨质谱(HRMS)等方法对化合物结构进行表征. 用四甲基偶氮唑盐(MTT)法评价了 该类化合物对人前列腺癌细胞(PC-3)、人食管癌细胞(EC-109)和人胃癌细胞(MGC-803)三种肿瘤细胞的抑制活性. 结果 显示, $N$-芐基-2-((4-甲基- $2 H$-色烯-2-酮-7-基)氧基)- $N$-(3,4,5-三甲氧基苯基)乙酰胺 (4a)和 $N$-((5-氯苯并 $[b]$ 富吩-3-基)甲 基)-2-((4-甲基- $2 \mathrm{H}$-色烯-2-酮-7-基)氧基)- $\mathrm{N}$-(3,4,5-三甲氧基苯基)乙酰胺(4n)对三种肿瘤细胞的抑制活性优于阳性对照药 5 -氟尿嘧啶，其中化合物 $\mathbf{4 n}$ 对人前列腺癌细胞( $\mathrm{PC}-3$ )的抑制活性最好，其 $\mathrm{IC}_{50}$ 为 $4.18 \mu \mathrm{mol} / \mathrm{L}$.
\end{abstract}

关键词 香豆素; 抗肿瘤; 合成

\section{Synthesis and Anticancer Activity of Novel Coumarin Derivatives}

\author{
Shi, Lei ${ }^{a}$ \\ Li, Ziqiu ${ }^{a}$ \\ Cui, Xinxin ${ }^{b}$ \\ Zhu, Ting ${ }^{b}$ \\ Pang, Xiaojing ${ }^{b}$ \\ Li, Longhui ${ }^{a}$ \\ Luo, Defu ${ }^{a}$ \\ Liu, Fangfang ${ }^{b} \quad$ Zhao, Bingyu $^{c}$ \\ Long, Yue*,a \\ Zhang, Saiyang ${ }^{*, c}$ \\ ( ${ }^{a}$ College of Chemistry, Zhengzhou University, Zhengzhou 450001) \\ ( ${ }^{b}$ School of Pharmaceutical Sciences, Zhengzhou 450001) \\ ( ${ }^{c}$ School of Basic Medical Sciences, Zhengzhou University, Zhengzhou 450001)
}

\begin{abstract}
Nineteen novel 3,4,5-trimethoxyphenyl coumarin derivatives have been synthesized and evaluated for antitumor activity against three human cancer cell lines (EC-109, PC-3 and MGC-803). These chemical structures were well characterized by NMR and HRMS spectroscopic methods. $N$-Benzyl-2-((4-methyl-2-oxo-2H-chromen-7-yl)oxy)- $N$-(3,4,5-trimethoxyphenyl)acetamide) (4a) and $N$-((5-chlorobenzo[b]thiophen-3-yl)methyl)-2-((4-methyl-2-oxo- $H$-chromen-7-yl)oxy)- $N$-(3,4,5trimethoxyphenyl)acetamide) (4n) had better inhibitory activity against three kinds of tumor cells than 5-fluorouracil. Compound $4 \mathrm{n}$ showed the most potent antitumor activity against PC-3 cells with an $\mathrm{IC}_{50}$ value of $4.18 \mu \mathrm{mol} / \mathrm{L}$.

Keywords coumarin; anticancer; synthesis
\end{abstract}

香豆素又称 1,2-苯并吡喃酮, 其结构中的苯环和吡 喃酮环可形成一个较大的共轭体系，这种结构特征使得 香豆素具有很强的可修饰性, 能够引入多种功能性基 团 ${ }^{[1]}$, 使其在生物医药、功能材料和光电材料等领域广 泛应用 ${ }^{[2]}$. 此外, 香豆素类化合物具有多种生物活性, 如抗炎 ${ }^{[3]}$ 、抗病毒 ${ }^{[4]}$ 、抗菌 ${ }^{[3]}$ 、抗氧化 ${ }^{[6]}$ 和抗肿瘤 ${ }^{[7 ~ 9]}$ 等. 近十几年来, 人们对香豆素类化合物的研究不断深入,
发现许多香豆素类化合物主要通过调控细胞周期、诱导 细胞调亡或对多种酶和信号通路产生影响等途径抑制 肿瘤细胞的增殖 ${ }^{[10,11]}$.

微管在细胞有丝分裂和生长发育过程中起着非常 重要的作用, 因此微管蛋白是抗癌药物研究中的一个重 要靶点. 研究发现, 多种含 3,4,5-三甲氧基苯基片段的 化合物通过抑制微管蛋白聚合来抑制肿瘤细胞的增

\footnotetext{
* Corresponding authors. E-mail: longyue@zzu.edu.cn; saiyangz@zzu.edu.cn

Received November 23, 2019; revised January 15, 2020; published online April 9, 2020.

Project supported by the National Natural Sciences Foundations of China (Nos. 81703541, 81673322) and the China Postdoctoral Science Foundation (No. 2018M632812).

国家自然科学基金(Nos. 81703541, 81673322)和中国博士后科学基金(No. 2018M632812)资助项目.
} 
殖 ${ }^{[2]}$. 如 2-氨基-4-(3,4,5-三甲氧基苯基)-5-芳基噻唑作 为微管蛋白聚合抑制剂, 对多药耐药肿瘤细胞表现出有 效的抑制活性 ${ }^{[13]} ; 3-(3,4,5$-三甲氧基苯胺基)苯并呋喃作 用于微管蛋白的秋水仙碱结合位点，从而有效抑制肿瘤 细胞的增殖 ${ }^{[14]}$.

酰胺类化合物具有抗菌 ${ }^{[15]}$ 、抗肿瘤 ${ }^{[16]}$ 和杀虫 ${ }^{[17]}$ 等生 物活性, 广泛应用于药物、材料和工业溶剂等方面. 酰 胺结构是蛋白质的基本结构, 具有低毒和生物利用度高 等优点, 因此在药物分子设计中常被用到 ${ }^{[18]}$. 如温倩雯 等 ${ }^{[19]}$ 合成一系列 4-(5H-嘧啶并 [5,4- $b$ 吲哚-2-基-氨基)苯 甲酰胺类化合物, 结果显示该类化合物对肿瘤细胞增殖 有明显抑制作用; 张明千等 ${ }^{[20]}$ 合成了一系列 1,3,4-塞二 唑酰胺类化合物, 发现部分化合物对人前列腺癌细胞 (PC-3)的抑制作用要优于阳性对照药达卡巴嗪.

在前期工作中我们设计合成了一系列含 3,4,5-三甲 氧基苯基的三级酰胺类化合物, 发现含有 4-甲基香豆素 基团的化合物有较好的抗肿瘤活性. 利用药物化学中的 拼合原理, 将 3,4,5-三甲氧基苯基和香豆素这两个药效 基团通过酰胺片段连接(图 1), 设计合成了 19 个目标化 合物, 并测试了该类化合物对人食管癌细胞 (EC$109)$ 、人胃癌细胞(MGC-803)和人前列腺癌细胞(PC-3) 的抑制作用, 探索其构效关系, 为此类化合物的进一步 研究提供参考.

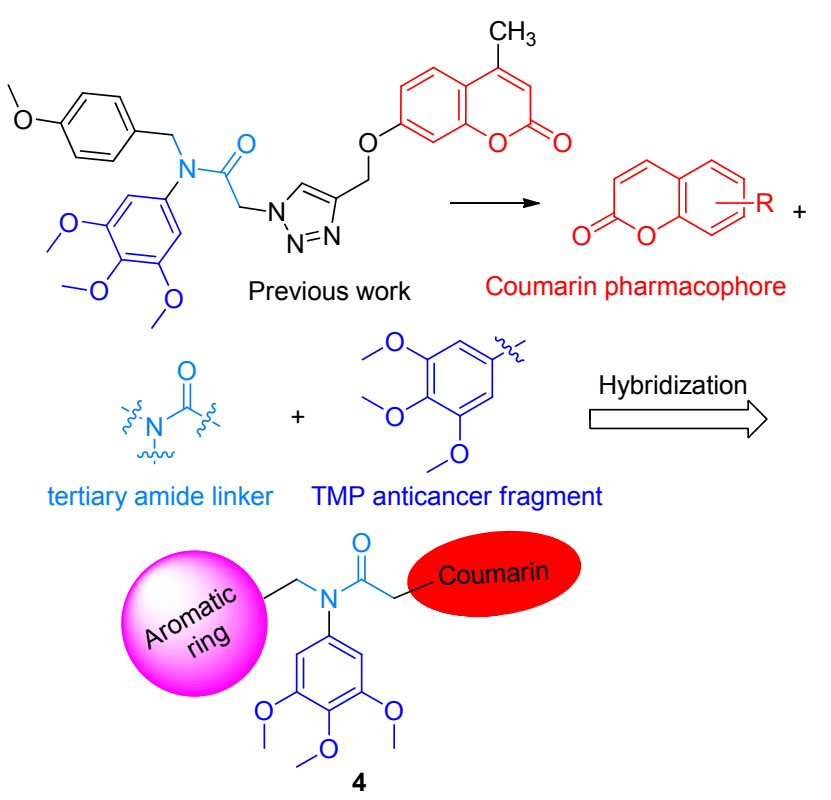

图 1 目标化合物 $\mathbf{4}$ 的分子设计示意图

Figure 1 Design strategy of the target compounds 4

\section{1 结果与讨论}

\section{1 化合物的合成}

3,4,5-三甲氧基苯胺和取代基苄氯在 $N, N$-二甲基甲
酰胺和碳酸钾作用下发生亲核取代反应生成化合物 $\mathbf{2}$, 再与氯乙酰氯反应得到化合物 3 , 最后, 再和香豆素通 过 Williamson 反应得到目标化合物 4. 在合成化合物 $\mathbf{2}$ 的过程中, 通常会有二取代副产物产生, 因此, 需要严 格控制反应物之间的物质的量比和反应时间. 参考文 献[21]中的合成方法，控制两种原料的物质的量比为 $1: 1$, 与参考文献不同的是没有对反应进行加热而是在 室温下进行. 实验过程中通过薄层色谱对反应进行监 测，当体系中出现二取代杂质点时停止反应，此时单取 代化合物 2 的收率最高. 最后一步反应的溶剂选择也非 常关键，参考文献[22]用丙酮作溶剂，发现完成反应需 要 $12 \mathrm{~h}$, 收率约为 $20 \%$. 为了提高收率缩短反应时间, 分别使用乙腈、乙醇和四氢呋喃等溶剂, 对比发现乙腈 作溶剂时, 反应时间缩短到 $5 \mathrm{~h}$, 大部分收率提高到 $60 \%$ 以上.

\section{2 化合物的抗肿瘤活性}

选用 5-氟尿嘧啶作阳性对照药, 用四甲基偶氮唑盐 (MTT)法评价该类化合物对人食管癌细胞(EC-109)、人 胃癌细胞(MGC-803)和人前列腺癌细胞(PC-3)三种肿瘤 细胞的抑制作用，其 $\mathrm{IC}_{50}$ 值见表 1.

从表 1 可以看出, 大部分化合物对肿瘤细胞具有一 定的抑制作用, 值得注意的是, 化合物 $4 \mathrm{a}$ 和 $4 \mathrm{n}$ 对三种 肿瘤细胞的抑制作用均优于阳性对照药 5-氟尿嘧啶, 其 中, 化合物 4n 对人前列腺癌细胞(PC-3)的抑制活性最 强, 其 $\mathrm{IC}_{50}$ 值为 $4.18 \mu \mathrm{mol} / \mathrm{L}$. 化合物 $4 \mathrm{c}$ 对人食管癌细胞 (EC-109)具有选择性抑制作用, 其 $\mathrm{IC}_{50}$ 值为 $10.96 \mu \mathrm{mol} /$ $\mathrm{L}$; 化合物 $4 \mathbf{e}$ 对人胃癌细胞(MGC-803)具有选择性抑制 作用, 其 $\mathrm{IC}_{50}$ 值为 $14.65 \mu \mathrm{mol} / \mathrm{L}$. 对比化合物 $\mathbf{4 b} \sim \mathbf{4 j}$ 对 人前列腺癌细胞( $\mathrm{PC}-3$ )的抑制活性发现，取代基 $\mathrm{R}^{1}$ 的种 类和位置对化合物的抗肿瘤活性有明显的影响. $\mathrm{R}^{1}$ 为给 电子取代基 (4b 和 $4 c)$ 时的抑制活性优于 $\mathrm{R}^{1}$ 为吸电子取 代基(4d、4e 和 $4 f)$ 时的抑制活性, 其抑制活性顺序为 $\mathrm{OCH}_{3}>\mathrm{CH}_{3}>\mathrm{Br}>\mathrm{F}>\mathrm{Cl} ; \mathrm{R}^{1}$ 位于对位 (4b、4c、4d 和 $\mathbf{4 f}$ ) 时的抗肿瘤活性普遍优于位于间位 $(4 \mathrm{~g} 、 4 h 、 4 i$ 和 $4 j)$ 时 的抗肿瘤活性. 比较含有不同香豆素基团的化合物 $4 \mathrm{n} \sim 4 \mathrm{~s}$ 对三种肿瘤细胞的抑制作用发现，当香豆素基 团的 4 位上含有甲基 $(4 \mathrm{n}$ 和 $4 p)$ 时，化合物对人前列腺癌 细胞(PC-3)和人胃癌细胞(MGC-803)均有较好抑制的作 用.

\section{2 结论}

利用拼合原理，设计并合成了 19 个未见文献报道 的新型香豆素类衍生物. 用 MTT 法对合成的化合物进 行抗肿瘤活性笁选, 结果显示, 化合物 $\mathbf{4 a}$ 和 $4 \mathrm{n}$ 对三种 肿瘤细胞的抑制作用均优于阳性对照药 5-氟尿嘧啶. 此 


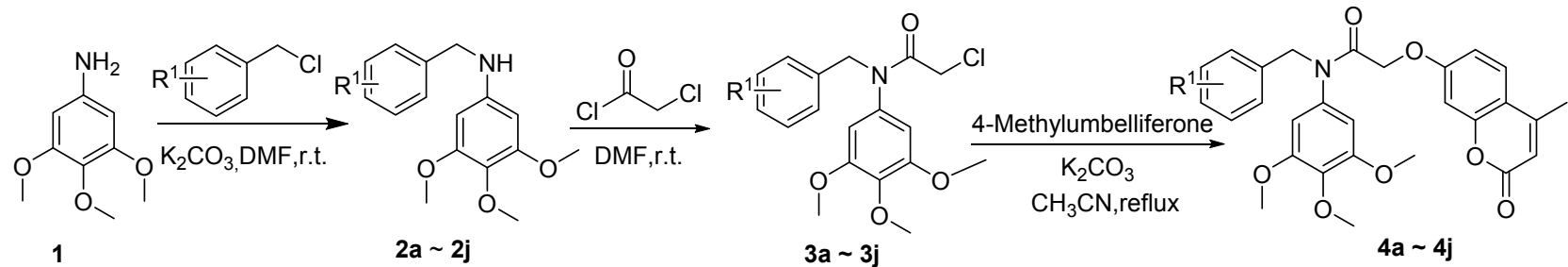

4a: $\mathrm{R}^{1}=4-\mathrm{H} ; 4 \mathrm{~b}: \mathrm{R}^{1}=4-\mathrm{OCH}_{3} ; 4 \mathrm{c}: \mathrm{R}^{1}=4-\mathrm{CH}_{3}$; 4d: $\mathrm{R}^{1}=4-\mathrm{F} ; \mathbf{4 e}: \mathrm{R}^{1}=4-\mathrm{Br} ; \mathbf{4 f}: \mathrm{R}^{1}=4-\mathrm{Cl} ; \mathbf{4 g}: \mathrm{R}^{1}=$ $3-\mathrm{OCH}_{3} ; \mathbf{4 h}: \mathrm{R}^{1}=3-\mathrm{CH}_{3} ; 4 \mathrm{i}: \mathrm{R}^{1}=3-\mathrm{F} ; \mathbf{4 j}: \mathrm{R}^{1}=3-\mathrm{Cl}$<smiles>[R]CNc1cc(OC)c(OC)c(OC)c1</smiles><smiles>[R]CN(C(=O)COc1ccc2c(C)cc(=O)oc2c1)C(=O)COc1cc(N(C)C)cc(OC)c1OC</smiles>
$2 k \sim 2 n$ $3 \mathbf{k} \sim 3 \mathbf{n}$ $4 k \sim 4 n$

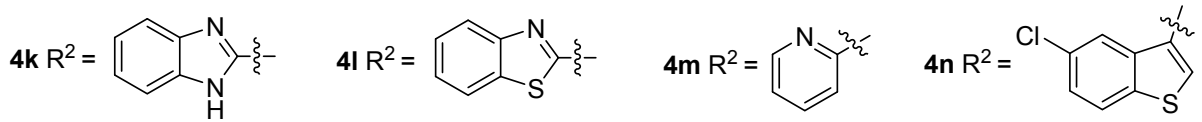<smiles>COc1cc(N(Cc2csc3ccc(Cl)cc23)C(=O)CCl)cc(OC)c1OC</smiles>
$3 n$<smiles></smiles><smiles>[R19]Cc1cc(C)cc2oc(=O)cc(C)c12</smiles>

图式 1 目标化合物 4 的合成路线

Scheme 1 Synthetic routes of the target compounds 4

表 1 化合物 $\mathbf{4 a} \sim \mathbf{4 s}$ 的抗肿瘤活性 $\left[\mathrm{IC}_{50} /\left(\mu \mathrm{mol} \cdot \mathrm{L}^{-1}\right)\right]$

Table 1 Anti-cancer activity $\left[\mathrm{IC}_{50}\left(\mu \mathrm{mol} \cdot \mathrm{L}^{-1}\right)\right]$ of the compounds $\mathbf{4 a} \sim \mathbf{4 s}$

\begin{tabular}{|c|c|c|c|}
\hline Compd. & PC-3 & EC-109 & MGC-803 \\
\hline $4 a$ & $5.62 \pm 1.27$ & $16.24 \pm 1.53$ & $18.90 \pm 1.28$ \\
\hline $4 b$ & $14.24 \pm 1.55$ & $38.73 \pm 0.42$ & $43.88 \pm 1.27$ \\
\hline $4 c$ & $31.51 \pm 2.94$ & $10.96 \pm 1.24$ & $>80$ \\
\hline $4 d$ & $45.58 \pm 2.02$ & $43.24 \pm 2.18$ & $>80$ \\
\hline $4 e$ & $32.91 \pm 1.81$ & $44.94 \pm 3.06$ & $14.65 \pm 1.82$ \\
\hline 4f & $>80$ & $>80$ & $>80$ \\
\hline $4 g$ & $76.28 \pm 2.59$ & $46.31 \pm 1.25$ & $78.29 \pm 3.64$ \\
\hline $4 h$ & $>80$ & $36.183 \pm 1.72$ & $>80$ \\
\hline $4 \mathbf{i}$ & $47.93 \pm 1.30$ & $27.93 \pm 1.30$ & $56.94 \pm 1.52$ \\
\hline $4 j$ & $>80$ & $72.86 \pm 1.37$ & $67.61 \pm 1.32$ \\
\hline $4 k$ & $12.81 \pm 1.63$ & $55.68 \pm 3.09$ & $>80$ \\
\hline 41 & $42.46 \pm 2.42$ & $51.33 \pm 3.82$ & $33.32 \pm 7.06$ \\
\hline $4 m$ & $39.33 \pm 2.02$ & $46.75 \pm 0.64$ & $76.19 \pm 2.42$ \\
\hline $4 n$ & $4.18 \pm 0.41$ & $14.32 \pm 2.48$ & $17.92 \pm 2.38$ \\
\hline 40 & $14.62 \pm 1.51$ & $42.93 \pm 2.04$ & $>80$ \\
\hline $4 p$ & $9.72 \pm 1.28$ & $24.19 \pm 1.82$ & $20.19 \pm 1.84$ \\
\hline $4 q$ & $42.18 \pm 1.39$ & $>80$ & $47.17 \pm 1.24$ \\
\hline $4 r$ & $32.06 \pm 1.59$ & $48.91 \pm 2.08$ & $>80$ \\
\hline $4 s$ & $>80$ & $60.63 \pm 3.47$ & $>80$ \\
\hline $5-\mathrm{Fu}$ & $9.79 \pm 0.17$ & $20.42 \pm 1.83$ & $21.21 \pm 3.61$ \\
\hline
\end{tabular}


外，化合物 4c 对人食管癌细胞(EC-109)的抑制作用以及 化合物 $4 \mathrm{e}$ 和 $4 \mathrm{p}$ 对人胃癌细胞(MGC-803)的抑制作用也 都优于阳性对照药 5-氟尿嘧啶.

\section{3 实验部分}

\section{1 仪器与试剂}

ZF7 三用紫外分析仪(巩义市予华仪器有限责任有 限公司); SHB-III 循环水式多用真空泵(郑州长城科工贸 有限公司); CP214 电子天平(奥豪斯仪器有限公司); RE5299 旋转蒸发仪 (上海亚荣生化仪器厂); Q-TOF Micro 高分辨质谱测定仪(美国 Waters 公司); DPX-400FT 超 导核磁共振仪(美国 Bruker 公司). 所用试剂均为市售分 析纯，使用前按常规方法处理. 实验所用的肿瘤细胞: 人食管癌细胞(EC-109)、人胃癌细胞(MGC-803)和人前 列腺癌细胞(PC-3)由郑州大学基础医学院药理系提供.

\section{2 实验方法}

\subsection{1 中间体 2 的合成}

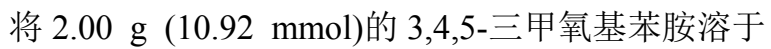
$\mathrm{N}, \mathrm{N}$-二甲基甲酰胺中, 加入取代基茮氯(10.92 mmol)和 $1.50 \mathrm{~g}(10.92 \mathrm{mmol})$ 的碳酸钾, 室温搅拌, 通过薄层色谱 (TLC)监测. 反应结束后抽滤, 收集滤液, 加入水和乙酸 乙酯萃取三次, 合并有机相, 无水硫酸镁干燥后旋干, 柱层析分离(洗脱剂: 石油醚-乙酸乙酯, $V: V=6: 1$ ), 得到目标化合物 $\mathbf{2 a} \sim \mathbf{2 n}$.

$N$-芐基-3,4,5-三甲氧基苯胺 (2a): 白色固体, 收率 $49 \%$. m.p. $84 \sim 85{ }^{\circ} \mathrm{C} ;{ }^{1} \mathrm{H}$ NMR (400 MHz, DMSO- $d_{6}$ ) $\delta$ : 7.37 (d, $J=7.0 \mathrm{~Hz}, 2 \mathrm{H}), 7.32(\mathrm{t}, J=7.5 \mathrm{~Hz}, 2 \mathrm{H}), 7.22(\mathrm{t}$, $J=7.2 \mathrm{~Hz}, 1 \mathrm{H}), 6.02(\mathrm{t}, J=6.0 \mathrm{~Hz}, 1 \mathrm{H}), 5.89(\mathrm{~s}, 2 \mathrm{H}), 4.23$ (d, $J=6.0 \mathrm{~Hz}, 2 \mathrm{H}), 3.64(\mathrm{~s}, 6 \mathrm{H}), 3.50(\mathrm{~s}, 3 \mathrm{H}) ;{ }^{13} \mathrm{C} \mathrm{NMR}$ $\left(100 \mathrm{MHz}, \mathrm{DMSO}-d_{6}\right) \delta: 153.3,145.4,140.4,128.5,128.2$, 127.4, 126.6, 90.1, 60.1, 55.4, 46.9; HRMS calcd for $\mathrm{C}_{16} \mathrm{H}_{20} \mathrm{NO}_{3}[\mathrm{M}+\mathrm{H}]^{+}$274.1443, found 274.1448.

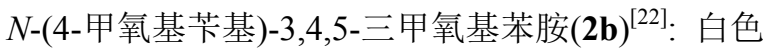
固体, 收率 58\%. m.p. 101 102 ${ }^{\circ} \mathrm{C} ;{ }^{1} \mathrm{H}$ NMR $(400 \mathrm{MHz}$, $\left.\mathrm{CDCl}_{3}\right) \delta: 7.23(\mathrm{~d}, J=8.6 \mathrm{~Hz}, 2 \mathrm{H}), 6.81(\mathrm{~d}, J=8.7 \mathrm{~Hz}$, $2 \mathrm{H}), 5.82(\mathrm{~s}, 2 \mathrm{H}), 4.15(\mathrm{~s}, 2 \mathrm{H}), 3.73(\mathrm{~s}, 3 \mathrm{H}), 3.72(\mathrm{~s}, 6 \mathrm{H})$, 3.69 (s, $3 \mathrm{H}) ;{ }^{13} \mathrm{C}$ NMR (100 MHz, DMSO- $\left.d_{6}\right) \delta: 158.1$, 153.3, 145.1, 131.9, 130.4, 128.7, 113.6, 90.4, 60.1, 55.4, 55.0, 46.5; HRMS calcd for $\mathrm{C}_{17} \mathrm{H}_{22} \mathrm{NO}_{4}[\mathrm{M}+\mathrm{H}]^{+}$ 304.1549 , found 304.1553 .

$N$-(4-甲基芐基)-3,4,5-三甲氧基苯胺(2c)：白色固体， 收率 54\%. m.p. 78 79 ${ }^{\circ} \mathrm{C} ;{ }^{1} \mathrm{H}$ NMR (400 MHz, DMSO$\left.d_{6}\right) \delta: 7.25(\mathrm{~d}, J=7.9 \mathrm{~Hz}, 2 \mathrm{H}), 7.12(\mathrm{~d}, J=7.8 \mathrm{~Hz}, 2 \mathrm{H})$, $5.96(\mathrm{t}, J=6.0 \mathrm{~Hz}, 1 \mathrm{H}), 5.88(\mathrm{~s}, 2 \mathrm{H}), 4.17(\mathrm{~d}, J=6.0 \mathrm{~Hz}$, $2 \mathrm{H}), 3.64(\mathrm{~s}, 6 \mathrm{H}), 3.50(\mathrm{~s}, 3 \mathrm{H}), 2.27(\mathrm{~s}, 3 \mathrm{H}) ;{ }^{13} \mathrm{C} \mathrm{NMR}$
(100 MHz, DMSO- $\left.d_{6}\right) \delta: 153.3,145.4,137.3,135.6,128.8$, 128.6, 127.3, 90.2, 60.1, 55.4, 46.7, 20.6; HRMS calcd for $\mathrm{C}_{17} \mathrm{H}_{22} \mathrm{NO}_{3}[\mathrm{M}+\mathrm{H}]^{+}$288.1600, found 288.1602.

$N$-(4-氟芐基)-3,4,5-三甲氧基苯胺(2d)：白色固体， 收率 46\%. m.p. $115 \sim 116{ }^{\circ} \mathrm{C}$; ${ }^{1} \mathrm{H}$ NMR $(400 \mathrm{MHz}$, DMSO- $\left.d_{6}\right) \delta: 7.40(\mathrm{dd}, J=8.6,5.7 \mathrm{~Hz}, 2 \mathrm{H}), 7.14(\mathrm{t}, J=8.9$ $\mathrm{Hz}, 2 \mathrm{H}), 6.08$ (s, 1H), 5.88 (s, 2H), 4.22 (s, 2H), 3.64 (s, $6 \mathrm{H}), 3.50(\mathrm{~s}, 3 \mathrm{H}) ;{ }^{13} \mathrm{C}$ NMR (100 MHz, DMSO- $\left.d_{6}\right) \delta$ : $162.3,159.9,158.7,153.3,147.0,144.6,98.7,90.6,60.1$, 55.9, 55.4; HRMS calcd for $\mathrm{C}_{16} \mathrm{H}_{19} \mathrm{FNO}_{3}[\mathrm{M}+\mathrm{H}]^{+}$ 292.1349 , found 292.1354

$\mathrm{N}$-(4-溴苄基)-3,4,5-三甲氧基苯胺 (2e)：灰色固体, 收率 55\%. m.p. 102 103 ${ }^{\circ} \mathrm{C} ;{ }^{1} \mathrm{H}$ NMR $(400 \mathrm{MHz}$, DMSO- $\left.d_{6}\right) \delta: 7.51(\mathrm{~d}, J=8.4 \mathrm{~Hz}, 2 \mathrm{H}), 7.33(\mathrm{~d}, J=8.4 \mathrm{~Hz}$, 2H), 6.09 (t, $J=6.1 \mathrm{~Hz}, 1 \mathrm{H}), 5.86(\mathrm{~s}, 2 \mathrm{H}), 4.21$ (d, $J=6.1$ $\mathrm{Hz}, 2 \mathrm{H}), 3.64$ (s, 6H), 3.50 (s, 3H); ${ }^{13} \mathrm{C}$ NMR (100 MHz, DMSO- $\left.d_{6}\right) \delta: 153.3,145.1,140.0,131.1,129.5,128.7$, 119.5, 90.2, 60.1, 55.5, 46.1; HRMS calcd for $\mathrm{C}_{16} \mathrm{H}_{18} \mathrm{Br}-$ $\mathrm{NO}_{3} \mathrm{Na}[\mathrm{M}+\mathrm{Na}]^{+} 374.0368$, found 374.0365.

$N$-(4-氯苄基)-3,4,5-三甲氧基苯胺 (2f): 灰色固体, 收率 53\%. m.p. 94 95 ${ }^{\circ} \mathrm{C} ;{ }^{1} \mathrm{H}$ NMR (400 MHz, DMSO$\left.d_{6}\right) \delta: 7.48 \sim 7.27(\mathrm{~m}, 4 \mathrm{H}), 6.08(\mathrm{t}, J=6.1 \mathrm{~Hz}, 1 \mathrm{H}), 5.86(\mathrm{~s}$, $2 \mathrm{H}), 4.23(\mathrm{~d}, J=6.0 \mathrm{~Hz}, 2 \mathrm{H}), 3.64(\mathrm{~s}, 6 \mathrm{H}), 3.50(\mathrm{~s}, 3 \mathrm{H})$; ${ }^{13} \mathrm{C}$ NMR (100 MHz, DMSO- $\left.d_{6}\right) \delta: 153.3,131.1,130.1$, 129.2, 128.9, 128.1, 98.8, 90.5, 60.1, 55.9, 55.5, 46.3; HRMS calcd for $\mathrm{C}_{16} \mathrm{H}_{18} \mathrm{ClNO}_{3} \mathrm{Na}[\mathrm{M}+\mathrm{Na}]^{+} 330.0873$, found 330.0876 .

$\mathrm{N}$-(3-甲氧基苄基)-3,4,5-三甲氧基苯胺 (2g)：白色固 体, 收率 49\%. m.p. 90 91 ${ }^{\circ} \mathrm{C} ;{ }^{1} \mathrm{H}$ NMR $(400 \mathrm{MHz}$, DMSO- $\left.d_{6}\right) \delta: 7.23(\mathrm{t}, J=8.0 \mathrm{~Hz}, 1 \mathrm{H}), 6.95(\mathrm{~d}, J=6.5 \mathrm{~Hz}$, 2H), 6.79 (dd, $J=8.1,1.8 \mathrm{~Hz}, 1 \mathrm{H}), 5.91(\mathrm{~s}, 2 \mathrm{H}), 4.20$ (s, $2 \mathrm{H}), 3.73$ (s, 3H), $3.65(\mathrm{~s}, 6 \mathrm{H}), 3.51$ (s, 3H); ${ }^{13} \mathrm{C}$ NMR $\left(100 \mathrm{MHz}\right.$, DMSO- $\left.d_{6}\right) \delta: 159.3,153.3,144.9,141.8,129.3$, 119.7, 113.1, 112.0, 98.7, 90.6, 60.1, 55.4, 54.9, 47.1; HRMS calcd for $\mathrm{C}_{17} \mathrm{H}_{22} \mathrm{NO}_{4}[\mathrm{M}+\mathrm{H}]^{+} 304.1549$, found 304.1555 .

$N$-(3-甲基苄基)-3,4,5-三甲氧基苯胺(2h): 黄色油状 液体，收率 $52 \% .{ }^{1} \mathrm{H}$ NMR $\left(400 \mathrm{MHz}, \mathrm{DMSO}-d_{6}\right) \delta: 7.20$ (dd, $J=9.7,4.8 \mathrm{~Hz}, 2 \mathrm{H}), 7.16(\mathrm{~d}, J=7.4 \mathrm{~Hz}, 1 \mathrm{H}), 7.04$ (d, $J=7.1 \mathrm{~Hz}, 1 \mathrm{H}), 5.97(\mathrm{t}, J=5.9 \mathrm{~Hz}, 1 \mathrm{H}), 5.90(\mathrm{~d}, J=8.2$ $\mathrm{Hz}, 2 \mathrm{H}), 4.18(\mathrm{~d}, J=5.8 \mathrm{~Hz}, 2 \mathrm{H}), 3.64(\mathrm{~s}, 6 \mathrm{H}), 3.50$ (s, $3 \mathrm{H}), 2.29$ (s, 3H); ${ }^{13} \mathrm{C}$ NMR (100 MHz, DMSO- $\left.d_{6}\right) \delta$ : $153.3,145.3,140.2,137.2,128.1,128.0,127.3,124.5$, 90.2, 60.1, 55.9, 55.4, 47.0, 21.0; HRMS calcd for $\mathrm{C}_{17} \mathrm{H}_{22} \mathrm{NO}_{3}[\mathrm{M}+\mathrm{H}]^{+}$288.1600, found 288.1603. 
$N$-(3-氟苄基)-3,4,5-三甲氧基苯胺 (2ii): 黄色固体, 收率 60\%. m.p. $75 \sim 76{ }^{\circ} \mathrm{C} ;{ }^{1} \mathrm{H}$ NMR $(400 \mathrm{MHz}$, DMSO$\left.d_{6}\right) \delta: 7.36(\mathrm{td}, J=7.9,6.3 \mathrm{~Hz}, 1 \mathrm{H}), 7.27 \sim 7.11(\mathrm{~m}, 2 \mathrm{H})$, 7.04 (td, $J=8.4,2.3 \mathrm{~Hz}, 1 \mathrm{H}), 6.10$ (t, $J=6.2 \mathrm{~Hz}, 1 \mathrm{H}), 5.88$ (s, 2H), 4.26 (d, $J=6.1 \mathrm{~Hz}, 2 \mathrm{H}), 3.64$ (s, 6H), 3.50 (s, 3H); ${ }^{13} \mathrm{C}$ NMR (100 MHz, DMSO- $d_{6}$ ) $\delta: 159.7,153.3,153.2$, $145.0,141.4,137.0,135.7,129.7,129.6,129.4,128.8$, 128.6, 127.4, 98.6, 90.4, 60.1, 55.4, 46.9; HRMS calcd for $\mathrm{C}_{16} \mathrm{H}_{18} \mathrm{FNO}_{3} \mathrm{Na}[\mathrm{M}+\mathrm{Na}]^{+}$292.1349, found 292.1353.

$N$-(3-氯芐基)-3,4,5-三甲氧基苯胺 (2j j): 黄色油状液 体，收率 43\%. ${ }^{1} \mathrm{H}$ NMR (400 MHz, DMSO- $d_{6}$ ) $\delta: 7.43$ (s, $1 \mathrm{H}), 7.35$ (dd, $J=3.4,2.0 \mathrm{~Hz}, 2 \mathrm{H}), 7.31 \sim 7.26(\mathrm{~m}, 1 \mathrm{H})$, $6.12(\mathrm{~s}, 1 \mathrm{H}), 5.88(\mathrm{~s}, 2 \mathrm{H}), 4.26(\mathrm{~s}, 2 \mathrm{H}), 3.65(\mathrm{~s}, 6 \mathrm{H}), 3.51$ $(\mathrm{s}, 3 \mathrm{H}) ;{ }^{13} \mathrm{C}$ NMR $\left(100 \mathrm{MHz}\right.$, DMSO- $\left.d_{6}\right) \delta: 153.3,133.0$, 130.1, 127.1, 126.6, 126.1, 98.9, 90.4, 60.1, 55.9, 55.4, 46.3; HRMS calcd for $\mathrm{C}_{16} \mathrm{H}_{19} \mathrm{ClNO}_{3}[\mathrm{M}+\mathrm{H}]^{+} 330.0873$, found 330.0874

$N$-((1H-苯并 $[d]$ 咪唑-2-基)甲基)-3,4,5-三甲氧基苯 胺(2k): 黄色固体, 收率 51\%. m.p. 50 52 ${ }^{\circ} \mathrm{C} ;{ }^{1} \mathrm{H}$ NMR $\left(400 \mathrm{MHz}, \mathrm{DMSO}-d_{6}\right) \delta: 12.29(\mathrm{~s}, 1 \mathrm{H}), 7.50$ (dd, $J=5.9$, $3.2 \mathrm{~Hz}, 2 \mathrm{H}), 7.13(\mathrm{dd}, J=6.0,3.2 \mathrm{~Hz}, 2 \mathrm{H}), 6.10(\mathrm{~s}, 1 \mathrm{H})$, 5.99 (s, 2H), 4.46 (s, 2H), 3.65 (s, 6H), $3.50(\mathrm{~s}, 3 \mathrm{H}) ;{ }^{13} \mathrm{C}$ NMR (100 MHz, DMSO- $\left.d_{6}\right) \delta: 153.6,153.3,145.0,129.0$, 121.4, 90.4, 60.1, 55.5, 42.1; HRMS calcd for $\mathrm{C}_{17} \mathrm{H}_{20} \mathrm{~N}_{3} \mathrm{O}_{3}$ $[\mathrm{M}+\mathrm{H}]^{+}$314.1505, found 314.1508.

$N$-(苯并 $[d]$ 塞唑-2-基甲基)-3,4,5-三甲氧基苯胺(21): 黄色油状液体, 收率 53\%. ${ }^{1} \mathrm{H}$ NMR (400 MHz, DMSO$\left.d_{6}\right) \delta: 8.03(\mathrm{~d}, J=7.9 \mathrm{~Hz}, 1 \mathrm{H}), 7.95(\mathrm{~d}, J=8.1 \mathrm{~Hz}, 1 \mathrm{H})$, 7.49 (t, $J=7.6 \mathrm{~Hz}, 1 \mathrm{H}), 7.40$ (t, $J=7.2 \mathrm{~Hz}, 1 \mathrm{H}), 6.52$ (s, $1 \mathrm{H}), 5.98(\mathrm{~s}, 2 \mathrm{H}), 4.70(\mathrm{~d}, J=5.2 \mathrm{~Hz}, 2 \mathrm{H}), 3.65(\mathrm{~s}, 6 \mathrm{H})$, $3.52(\mathrm{~s}, 3 \mathrm{H}) ;{ }^{13} \mathrm{C}$ NMR (100 MHz, DMSO- $\left.d_{6}\right) \delta: 174.6$, $153.4,153.0,144.5,134.6,129.2,126.0,124.8,122.3$, 122.2, 90.4, 60.1, 55.5, 45.9; HRMS calcd for $\mathrm{C}_{17} \mathrm{H}_{19} \mathrm{~N}_{2-}$ $\mathrm{O}_{3} \mathrm{~S}[\mathrm{M}+\mathrm{H}]^{+}$331.1116, found 331.1124.

$N$-(吡啶-2-基甲基)-3,4,5-三甲氧基苯胺 (2m): 黄色 油状液体, 收率 57\%. ${ }^{1} \mathrm{H}$ NMR (400 MHz, DMSO- $\left.d_{6}\right) \delta$ : $8.59 \sim 8.44(\mathrm{~m}, 1 \mathrm{H}), 7.74(\mathrm{td}, J=7.7,1.8 \mathrm{~Hz}, 1 \mathrm{H}), 7.38(\mathrm{~d}$, $J=7.8 \mathrm{~Hz}, 1 \mathrm{H}), 7.25(\mathrm{dd}, J=6.9,5.4 \mathrm{~Hz}, 1 \mathrm{H}), 6.13(\mathrm{t}$, $J=6.0 \mathrm{~Hz}, 1 \mathrm{H}), 5.90(\mathrm{~s}, 2 \mathrm{H}), 4.33(\mathrm{~d}, J=6.0 \mathrm{~Hz}, 2 \mathrm{H}), 3.64$ (s, 6H), $3.50(\mathrm{~s}, 3 \mathrm{H}) ;{ }^{13} \mathrm{C}$ NMR (100 MHz, DMSO- $\left.d_{6}\right) \delta$ : $159.8,153.4,148.7,145.1,136.6,128.7,122.0,121.3$, 90.2, 60.1, 55.4, 48.9; HRMS calcd for $\mathrm{C}_{15} \mathrm{H}_{19} \mathrm{~N}_{2} \mathrm{O}_{3}[\mathrm{M}+$ $\mathrm{H}]^{+}$275.1396, found 275.1398.

$N$-((5-氯苯并 $[b]$ 噻吩-3-基)甲基)-3,4,5-三甲氧基苯 胺(2n): 白色固体, 收率 $44 \%$. m.p. $140 \sim 141{ }^{\circ} \mathrm{C} ;{ }^{1} \mathrm{H}$
NMR (400 MHz, DMSO- $\left.d_{6}\right) \delta: 8.06(\mathrm{~d}, J=1.9 \mathrm{~Hz}, 1 \mathrm{H})$, 8.02 (d, $J=8.6 \mathrm{~Hz}, 1 \mathrm{H}), 7.77$ (s, 1H), 7.41 (dd, $J=8.6,2.0$ $\mathrm{Hz}, 1 \mathrm{H}), 6.05$ (t, $J=5.8 \mathrm{~Hz}, 1 \mathrm{H}), 5.99$ (s, 2H), 4.47 (d, $J=$ $5.8 \mathrm{~Hz}, 2 \mathrm{H}), 3.68(\mathrm{~s}, 6 \mathrm{H}), 3.51(\mathrm{~s}, 3 \mathrm{H}) ;{ }^{13} \mathrm{C}$ NMR $(100$ MHz, DMSO- $d_{6}$ ) $\delta$ : 153.4, 145.2, 139.5, 138.5, 134.3, $129.3,128.7,126.3,124.5,124.4,121.8,90.2,60.1,55.5$, 41.3; HRMS calcd for $\mathrm{C}_{18} \mathrm{H}_{19} \mathrm{ClNO}_{3} \mathrm{~S}[\mathrm{M}+\mathrm{H}]^{+}$364.0774, found 364.0779 .

\subsection{2中间体 $\mathbf{3}$ 的合成}

将 $0.50 \mathrm{~g}(2.09 \mathrm{mmol})$ 的化合物 $\mathbf{2 a} \sim 2 \mathbf{n}$ 加入 $100 \mathrm{~mL}$ 的圆底烧瓶中, 用 $N, N$-二甲基甲酰胺溶解, 缓慢滴加氯 乙酰氯 $(2.51 \mathrm{mmol})$, 室温摚拌, TLC 检测反应完全后, 加入水和乙酸乙酯萃取三次, 合并有机相, 无水硫酸镁 干燥，旋干得化合物 $\mathbf{3 a} \sim \mathbf{3 n}$.

2 -氯- $N$ - 芐基- $N$-(3,4,5-三甲氧基苯基) 乙酰胺 (3a): 白色固体, 收率 73\%. m.p. 137 138 ${ }^{\circ} \mathrm{C} ;{ }^{1} \mathrm{H}$ NMR $(400$ $\left.\mathrm{MHz}, \mathrm{CDCl}_{3}\right) \delta: 7.22(\mathrm{~s}, 1 \mathrm{H}), 7.21(\mathrm{~d}, J=1.3 \mathrm{~Hz}, 2 \mathrm{H})$, $7.18 \sim 7.13(\mathrm{~m}, 2 \mathrm{H}), 6.09(\mathrm{~s}, 2 \mathrm{H}), 4.79(\mathrm{~s}, 2 \mathrm{H}), 3.83(\mathrm{~s}$, 2H), 3.77 (s, 3H), $3.62(\mathrm{~s}, 6 \mathrm{H}) ;{ }^{13} \mathrm{C}$ NMR $(100 \mathrm{MHz}$, $\left.\mathrm{CDCl}_{3}\right) \delta: 165.2,152.6,137.1,135.9,135.2,128.3,127.5$, $126.8,104.5,60.0,55.1,52.5,40.9$; HRMS calcd for $\mathrm{C}_{18} \mathrm{H}_{21} \mathrm{ClNO}_{4}[\mathrm{M}+\mathrm{H}]^{+} 350.1159$, found 350.1162 .

2-氯- $\mathrm{N}$-(4-甲氧基芐基)- $\mathrm{N}$-( $3,4,5$-三甲氧基苯基)乙 酰胺 $(3 \mathbf{b})$ : 白色固体, 收率 75\%. m.p. $136 \sim 137{ }^{\circ} \mathrm{C} ;{ }^{1} \mathrm{H}$ NMR (400 MHz, $\left.\mathrm{CDCl}_{3}\right) \delta: 7.08(\mathrm{~d}, J=8.7 \mathrm{~Hz}, 2 \mathrm{H}), 6.74$ (d, $J=8.7 \mathrm{~Hz}, 2 \mathrm{H}), 6.10$ (s, 2H), $4.72(\mathrm{~s}, 2 \mathrm{H}), 3.81$ (s, 2H), 3.78 (s, 3H), $3.71(\mathrm{~s}, 3 \mathrm{H}), 3.64(\mathrm{~s}, 6 \mathrm{H}) ;{ }^{13} \mathrm{C}$ NMR $(100$ $\left.\mathrm{MHz}, \mathrm{CDCl}_{3}\right) \delta: 165.1,158.3,152.6,137.2,135.2,129.7$, $128.1,112.8,104.6,60.0,55.2,54.3,52.0,40.9$; HRMS calcd for $\mathrm{C}_{19} \mathrm{H}_{23} \mathrm{ClNO}_{5}[\mathrm{M}+\mathrm{H}]^{+} 380.1265$, found 380.1270 .

2 -氯- $N$-(4-甲基苄基)- $N$-( $3,4,5$-三甲氧基苯基)乙酰 胺(3c): 白色固体, 收率 68\%. m.p. $143 \sim 144{ }^{\circ} \mathrm{C} ;{ }^{1} \mathrm{H}$ NMR (400 MHz, $\left.\mathrm{CDCl}_{3}\right) \delta: 7.05(\mathrm{~d}, J=8.3 \mathrm{~Hz}, 2 \mathrm{H}), 7.02$ (d, $J=8.3 \mathrm{~Hz}, 2 \mathrm{H}), 6.10$ (s, 2H), $4.74(\mathrm{~s}, 2 \mathrm{H}), 3.82(\mathrm{~s}, 2 \mathrm{H})$, $3.78(\mathrm{~s}, 3 \mathrm{H}), 3.64(\mathrm{~s}, 6 \mathrm{H}) ;{ }^{13} \mathrm{C}$ NMR $\left(100 \mathrm{MHz}, \mathrm{CDCl}_{3}\right) \delta$ : $165.1,152.6,137.2,136.5,135.3,132.9,128.2,128.1$, 104.6, 59.9, 55.2, 52.3, 40.9, 20.1; HRMS calcd for $\mathrm{C}_{19} \mathrm{H}_{23} \mathrm{ClNO}_{4}[\mathrm{M}+\mathrm{H}]^{+}$364.1316, found 364.1318.

2-氯- $N$-(4-氟苄基)- $N$-( $3,4,5$-三甲氧基苯基)乙酰胺 (3d): 白色固体, 收率 78\%. m.p. 119 121 ${ }^{\circ} \mathrm{C} ;{ }^{1} \mathrm{H}$ NMR (400 MHz, $\mathrm{CDCl}_{3}$ ) $\delta: 7.22(\mathrm{dd}, J=8.6,5.4 \mathrm{~Hz}, 2 \mathrm{H}), 6.98$ (t, $J=8.7 \mathrm{~Hz}, 2 \mathrm{H}), 6.17$ (s, 2H), $4.82(\mathrm{~s}, 2 \mathrm{H}), 3.89$ (s, 2H), $3.85(\mathrm{~s}, 3 \mathrm{H}), 3.72(\mathrm{~s}, 6 \mathrm{H}) ;{ }^{13} \mathrm{C} \mathrm{NMR}\left(100 \mathrm{MHz}, \mathrm{CDCl}_{3}\right) \delta$ : $165.2,162.6,160.2,152.7,137.2,135.0,131.8,131.7$, 
130.1, 130.0, 114.4, 114.2, 104.4, 60.0, 55.2, 51.8, 40.8; HRMS calcd for $\mathrm{C}_{18} \mathrm{H}_{20} \mathrm{ClFNO}_{4}[\mathrm{M}+\mathrm{H}]^{+}$368.1065, found 368.1072

2-氯- $N$-(4-溴苄基)- $N$-(3,4,5-三甲氧基苯基)乙酰胺 (3e): 白色固体, 收率 73\%. m.p. 169 $172{ }^{\circ} \mathrm{C} ;{ }^{1} \mathrm{H}$ NMR (400 MHz, DMSO- $d_{6}$ ) $\delta: 7.51$ (d, $\left.J=8.3 \mathrm{~Hz}, 2 \mathrm{H}\right), 7.20$ (d, $J=8.3 \mathrm{~Hz}, 2 \mathrm{H}), 6.56$ (s, 2H), 4.83 (s, 2H), 4.19 (s, 2H), $3.67(\mathrm{~d}, J=17.6 \mathrm{~Hz}, 9 \mathrm{H}) ;{ }^{13} \mathrm{C}$ NMR $\left(100 \mathrm{MHz}, \mathrm{DMSO}-d_{6}\right)$ $\delta: 165.7,153.0,137.1,136.9,136.5,136.0,131.2,130.4$, $120.4,105.8,60.0,56.0,52.1$; HRMS calcd for $\mathrm{C}_{18} \mathrm{H}_{20} \mathrm{Br}-$ $\mathrm{ClNO}_{4}[\mathrm{M}+\mathrm{H}]^{+}$428.0264, found 428.0269.

2-氯- $N$-(4-氯苄基)- $N$-(3,4,5-三甲氧基苯基)乙酰胺 (3f): 白色固体, 收率 79\%. m.p. 166 $167{ }^{\circ} \mathrm{C} ;{ }^{1} \mathrm{H}$ NMR $\left(400 \mathrm{MHz}, \mathrm{CDCl}_{3}\right) \delta: 7.27$ (d, $\left.J=2.8 \mathrm{~Hz}, 2 \mathrm{H}\right), 7.19$ (d, $J=$ $8.4 \mathrm{~Hz}, 2 \mathrm{H}), 6.18$ (s, 2H), $4.82(\mathrm{~s}, 2 \mathrm{H}), 3.90$ (s, 2H), 3.86 $(\mathrm{s}, 3 \mathrm{H}), 3.73(\mathrm{~s}, 6 \mathrm{H}) ;{ }^{13} \mathrm{C} \mathrm{NMR}\left(100 \mathrm{MHz}, \mathrm{CDCl}_{3}\right) \delta$ : $166.4,153.8,138.3,136.1,135.4,133.8,130.7,128.7$, 105.4, 61.0, 56.2, 53.0, 41.8; HRMS calcd for $\mathrm{C}_{18} \mathrm{H}_{20^{-}}$ $\mathrm{Cl}_{2} \mathrm{NO}_{4}[\mathrm{M}+\mathrm{H}]^{+}$384.0769, found 384.0773.

2-氯- $N$-(3-甲氧基苄基)- $N$-(3,4,5-三甲氧基苯基)乙 酰胺(3g): 白色固体, 收率 75\%. m.p. 143 144 ${ }^{\circ} \mathrm{C} ;{ }^{1} \mathrm{H}$ NMR (400 MHz, $\left.\mathrm{CDCl}_{3}\right) \delta: 7.13(\mathrm{t}, J=8.1 \mathrm{~Hz}, 1 \mathrm{H})$, $6.77 \sim 6.70(\mathrm{~m}, 3 \mathrm{H}), 6.13(\mathrm{~s}, 2 \mathrm{H}), 4.76(\mathrm{~s}, 2 \mathrm{H}), 3.84(\mathrm{~s}$, 2H), 3.78 (s, 3H), $3.69(\mathrm{~s}, 3 \mathrm{H}), 3.64(\mathrm{~s}, 6 \mathrm{H}) ;{ }^{13} \mathrm{C} \mathrm{NMR}$ $\left(100 \mathrm{MHz}, \mathrm{DMSO}-d_{6}\right) \delta: 161.0,154.5,148.4,133.1,133.0$, $131.0,124.2,116.3,109.3,108.2,100.3,55.7,50.9,50.0$, 48.3, 36.6; HRMS calcd for $\mathrm{C}_{19} \mathrm{H}_{22} \mathrm{ClNO}_{5} \mathrm{Na}[\mathrm{M}+\mathrm{Na}]^{+}$ 402.1084, found 402.1085 .

2-氯- $N$-(3-甲基苠基)- $N$-(3,4,5-三甲氧基苯基)乙酰 胺(3h): 白色固体, 收率 72\%. m.p. 93 95 ${ }^{\circ} \mathrm{C} ;{ }^{1} \mathrm{H}$ NMR $\left(400 \mathrm{MHz}, \mathrm{CDCl}_{3}\right) \delta: 7.10(\mathrm{t}, J=7.5 \mathrm{~Hz}, 1 \mathrm{H}), 7.04 \sim 6.97$ $(\mathrm{m}, 2 \mathrm{H}), 6.93(\mathrm{~d}, J=7.5 \mathrm{~Hz}, 1 \mathrm{H}), 6.11(\mathrm{~s}, 2 \mathrm{H}), 4.75$ (s, $2 \mathrm{H}), 3.84$ (s, 2H), 3.78 (s, 3H), $3.63(\mathrm{~s}, 6 \mathrm{H}), 2.23$ (s, 3H); ${ }^{13} \mathrm{C}$ NMR (100 MHz, $\left.\mathrm{CDCl}_{3}\right) \delta: 165.4,152.6,137.1,135.7$, 135.2, 129.0, 127.5, 127.3, 125.3, 104.5, 59.9, 55.1, 52.6, 40.9, 39.8, 20.3; HRMS calcd for $\mathrm{C}_{19} \mathrm{H}_{23} \mathrm{ClNO}_{4}[\mathrm{M}+\mathrm{H}]^{+}$ 364.1316 , found 364.1321 .

2-氯- $N$-(3-氟苄基)- $N$-(3,4,5-三甲氧基苯基) 乙酰胺 (3i): 白色固体, 收率 77\%. m.p. $110 \sim 112{ }^{\circ} \mathrm{C} ;{ }^{1} \mathrm{H}$ NMR $\left(400 \mathrm{MHz}, \mathrm{CDCl}_{3}\right) \delta: 7.22 \sim 7.17(\mathrm{~m}, 1 \mathrm{H}), 6.96(\mathrm{~s}, 1 \mathrm{H})$, $6.92(\mathrm{dd}, J=9.1,1.1 \mathrm{~Hz}, 2 \mathrm{H}), 6.13(\mathrm{~s}, 2 \mathrm{H}), 4.77(\mathrm{~s}, 2 \mathrm{H})$, $3.84(\mathrm{~s}, 2 \mathrm{H}), 3.78(\mathrm{~s}, 3 \mathrm{H}), 3.66(\mathrm{~s}, 6 \mathrm{H}) ;{ }^{13} \mathrm{C}$ NMR $(100$ $\left.\mathrm{MHz}, \mathrm{CDCl}_{3}\right) \delta: 165.4,163.0,160.5,152.7,138.4,138.3$, $137.3,135.1,129.03,129.0,123.8,123.8,115.1,114.9$, $113.8,113.6,104.3,60.0,55.2,52.1,40.8$; HRMS calcd for $\mathrm{C}_{18} \mathrm{H}_{20} \mathrm{ClFNO}_{4}[\mathrm{M}+\mathrm{H}]^{+}$368.1065, found 368.1066.

2-氯- $N$-(3-氯苄基)- $N$-(3,4,5-三甲氧基苯基) 乙酰胺 (3j): 白色固体, 收率 70\%. m.p. 85 86 ${ }^{\circ} \mathrm{C} ;{ }^{1} \mathrm{H}$ NMR $\left(400 \mathrm{MHz}, \mathrm{CDCl}_{3}\right) \delta: 7.19$ (d, J=1.6 Hz, 1H), 7.17 (s, $1 \mathrm{H}), 7.08$ (d, $J=6.8 \mathrm{~Hz}, 1 \mathrm{H}), 6.13$ (s, 2H), 4.75 (s, 2H), 3.84 (s, 2H), 3.79 (s, 3H), 3.67 (s, 6H); ${ }^{13} \mathrm{C}$ NMR (100 $\left.\mathrm{MHz}, \mathrm{CDCl}_{3}\right) \delta: 165.5,152.8,137.8,137.3,135.0,133.2$, 128.8, 128.3, 127.0, 126.4, 104.4, 60.0, 55.2, 52.1, 40.7; HRMS calcd for $\mathrm{C}_{18} \mathrm{H}_{19} \mathrm{Cl}_{2} \mathrm{NO}_{4} \mathrm{Na}[\mathrm{M}+\mathrm{Na}]^{+}$406.0589, found 406.0593 .

2-氯- $N$-((1H-苯并 $[d]$ 咪唑-2-基)甲基)- $N$-(3,4,5-三甲 氧基苯基)乙酰胺(3k)：黄色固体，收率 76\%. m.p. 134 $135{ }^{\circ} \mathrm{C} ;{ }^{1} \mathrm{H}$ NMR $\left(400 \mathrm{MHz}, \mathrm{DMSO}-d_{6}\right) \delta: 7.57$ (dd, $J=$ 6.0, 3.2 Hz, 2H), 7.23 (dd, $J=6.0,3.1 \mathrm{~Hz}, 2 \mathrm{H}), 6.90$ (s, $2 \mathrm{H}), 5.12(\mathrm{~s}, 2 \mathrm{H}), 4.26(\mathrm{~s}, 2 \mathrm{H}), 3.70(\mathrm{~s}, 6 \mathrm{H}), 3.64(\mathrm{~s}, 3 \mathrm{H})$; ${ }^{13} \mathrm{C}$ NMR (100 MHz, DMSO- $\left.d_{6}\right) \delta$ : 168.6, 165.8, 162.3, $153.0,150.3,137.2,136.1,121.9,105.9,59.9,55.9,47.4$, 43.0,41.5,35.8, 21.0; HRMS calcd for $\mathrm{C}_{19} \mathrm{H}_{20} \mathrm{ClN}_{3} \mathrm{O}_{4} \mathrm{Na}$ $[\mathrm{M}+\mathrm{Na}]^{+}$412.1040, found 412.1053.

2-氯- $N$-(苯并 $[d]$ 噻唑-2-基甲基)- $N$-(3,4,5-三甲氧基 苯基)乙酰胺(3I)：黄色固体，收率 70\%. m.p. 147 $148{ }^{\circ} \mathrm{C} ;{ }^{1} \mathrm{H}$ NMR (400 MHz, $\mathrm{CDCl}_{3}$ ) $\delta: 7.90$ (d, $J=8.1$ $\mathrm{Hz}, 1 \mathrm{H}), 7.82$ (d, $J=7.9 \mathrm{~Hz}, 1 \mathrm{H}), 7.41$ (dd, $J=11.2,4.1$ $\mathrm{Hz}, 1 \mathrm{H}), 7.34$ (dd, $J=11.1,4.1 \mathrm{~Hz}, 1 \mathrm{H}), 6.43$ (s, 2H), 5.22 (s, 2H), 3.92 (s, 2H), 3.77 (s, 3H), $3.68(\mathrm{~s}, 6 \mathrm{H}) ;{ }^{13} \mathrm{C}$ NMR $\left(100 \mathrm{MHz}, \mathrm{CDCl}_{3}\right) \delta: 165.8,165.6,152.9,151.3,137.5$, 135.1, 134.6, 125.3, 124.5, 121.9, 120.8, 104.1, 59.9, 55.2, 50.9, 40.3; HRMS calcd for $\mathrm{C}_{19} \mathrm{H}_{20} \mathrm{ClN}_{3} \mathrm{O}_{4}[\mathrm{M}+\mathrm{H}]^{+}$ 407.0832, found 407.0837.

2-氯- $N$-(吡啶-2-基甲基)- $N$-(3,4,5-三甲氧基苯基)乙 酰胺 $(3 \mathrm{~m})$ : 黄色固体，收率 71\%. m.p. $121 \sim 122{ }^{\circ} \mathrm{C} ;{ }^{1} \mathrm{H}$ NMR (400 MHz, $\left.\mathrm{CDCl}_{3}\right) \delta: 8.46(\mathrm{~d}, J=4.3 \mathrm{~Hz}, 1 \mathrm{H}), 7.63$ (td, $J=7.7,1.6 \mathrm{~Hz}, 1 \mathrm{H}), 7.36$ (d, $J=7.8 \mathrm{~Hz}, 1 \mathrm{H}), 7.15$ (dd, $J=6.8,5.2 \mathrm{~Hz}, 1 \mathrm{H}), 6.37$ (s, 2H), $4.96(\mathrm{~s}, 2 \mathrm{H}), 3.90(\mathrm{~s}$, 2H), 3.77 (s, 3H), $3.69(\mathrm{~s}, 6 \mathrm{H}) ;{ }^{13} \mathrm{C} \mathrm{NMR} \mathrm{(100} \mathrm{MHz,}$ $\left.\mathrm{CDCl}_{3}\right) \delta: 165.8,155.0,152.8,147.1,137.2,136.9,135.7$, 122.5, 121.9, 104.3, 59.9, 55.2, 53.9, 40.7; HRMS calcd for $\mathrm{C}_{17} \mathrm{H}_{20} \mathrm{ClN}_{2} \mathrm{O}_{4}[\mathrm{M}+\mathrm{H}]^{+}$351.1112, found 351.1108.

2 -氯- $N$-((5-氯苯并 $[b]$ 噻吩-3-基)甲基)- $N$-(3,4,5-三甲 氧基苯基)乙酰胺(3n): 白色固体，收率 67\%. m.p. 154 $156{ }^{\circ} \mathrm{C} ;{ }^{1} \mathrm{H}$ NMR (400 MHz, $\mathrm{CDCl}_{3}$ ) $\delta: 7.67$ (d, $J=8.6$ $\mathrm{Hz}, 1 \mathrm{H}), 7.59$ (d, $J=1.8 \mathrm{~Hz}, 1 \mathrm{H}), 7.25$ (d, $J=1.9 \mathrm{~Hz}, 1 \mathrm{H})$, $7.23(\mathrm{~d}, J=3.6 \mathrm{~Hz}, 1 \mathrm{H}), 6.04(\mathrm{~s}, 2 \mathrm{H}), 5.00(\mathrm{~s}, 2 \mathrm{H}), 3.82$ (s, $2 \mathrm{H}), 3.77(\mathrm{~s}, 3 \mathrm{H}), 3.56(\mathrm{~s}, 6 \mathrm{H}) ;{ }^{13} \mathrm{C} \mathrm{NMR}(100 \mathrm{MHz}$, $\left.\mathrm{CDCl}_{3}\right) \delta: 165.2,152.7,138.3,137.5,137.2,134.7,130.0$, 
$129.8,128.1,124.0,122.7,121.1,104.6,60.0,55.2,45.4$, 40.8; HRMS calcd for $\mathrm{C}_{20} \mathrm{H}_{20} \mathrm{Cl}_{2} \mathrm{NO}_{4} \mathrm{~S}[\mathrm{M}+\mathrm{H}]^{+}$ 440.0490 , found 440.0496 .

\subsection{3 目标化合物 4 的合成}

在 $50 \mathrm{~mL}$ 圆底烧瓶中加入 $0.20 \mathrm{~g}(0.48 \mathrm{mmol})$ 的化合 物 3, 不同取代香豆素 $(0.48 \mathrm{mmol})$, 碳酸钾 $(0.96 \mathrm{mmol})$ 和乙腈加热回流 $5 \mathrm{~h}$. 反应结束后, 冷却至室温, 抽滤得 滤液, 柱层析分离(洗脱剂: 石油醚-乙酸乙酯, $V: V=$ $4: 1)$, 得到目标产物 $\mathbf{4 a} \sim \mathbf{4 s}$.

$N$-芐基-2-((4-甲基-2 $\mathrm{H}$-色烯-2-酮-7-基)氧基)- $N$ (3,4,5-三甲氧基苯基)乙酰胺(4a): 白色固体，收率 59\%. m.p. $138 \sim 140{ }^{\circ} \mathrm{C} ;{ }^{1} \mathrm{H}$ NMR $\left(400 \mathrm{MHz}, \mathrm{CDCl}_{3}\right) \delta: 7.42$ $(\mathrm{d}, J=8.8 \mathrm{~Hz}, 1 \mathrm{H}), 7.22(\mathrm{~s}, 1 \mathrm{H}), 7.21(\mathrm{t}, J=3.2 \mathrm{~Hz}, 2 \mathrm{H})$, $7.16(\mathrm{dd}, J=6.6,2.7 \mathrm{~Hz}, 2 \mathrm{H}), 6.83(\mathrm{dd}, J=8.8,2.4 \mathrm{~Hz}$, $1 \mathrm{H}), 6.59$ (d, $J=2.4 \mathrm{~Hz}, 1 \mathrm{H}), 6.15 \sim 6.08(\mathrm{~m}, 2 \mathrm{H}), 6.07$ (d, $J=0.7 \mathrm{~Hz}, 1 \mathrm{H}), 4.80$ (s, 2H), 4.46 (s, 2H), 3.78 (d, $J=7.2$ $\mathrm{Hz}, 3 \mathrm{H}), 3.64$ (d, $J=10.8 \mathrm{~Hz}, 6 \mathrm{H}), 2.32$ (d, $J=0.6 \mathrm{~Hz}$, $3 \mathrm{H}) ;{ }^{13} \mathrm{C} \mathrm{NMR}\left(100 \mathrm{MHz}, \mathrm{CDCl}_{3}\right) \delta: 165.4,160.1,160.0$, $154.0,152.9,151.5,137.4,135.9,134.3,128.4,127.5$, 126.9, 124.6, 113.1, 112.2, 111.2, 104.4, 100.2, 65.1, 59.9, 55.2, 52.3, 17.7; HRMS calcd for $\mathrm{C}_{28} \mathrm{H}_{28} \mathrm{NO}_{7}[\mathrm{M}+\mathrm{H}]^{+}$ 490.1860 , found 490.1873 .

$N$-(4-甲氧基苄基)-2-((4-甲基- $2 H$-色烯-2-酮-7-基)氧 基)- $N$-(3,4,5-三甲氧基苯基)乙酰胺(4b): 白色固体, 收 率 77\%. m.p. 188 $190{ }^{\circ} \mathrm{C}$; ${ }^{1} \mathrm{H}$ NMR (400 MHz, $\mathrm{CDCl}_{3}$ ) $\delta: 7.42(\mathrm{~d}, J=8.8 \mathrm{~Hz}, 1 \mathrm{H}), 7.08$ (d, $J=8.6 \mathrm{~Hz}, 2 \mathrm{H}), 6.82$ (dd, $J=8.8,2.5 \mathrm{~Hz}, 1 \mathrm{H}), 6.75$ (d, $J=8.6 \mathrm{~Hz}, 2 \mathrm{H}), 6.58$ (d, $J=2.4 \mathrm{~Hz}, 1 \mathrm{H}), 6.12$ (s, 2H), 6.06 (d, $J=0.9 \mathrm{~Hz}, 1 \mathrm{H}), 4.73$ $(\mathrm{s}, 2 \mathrm{H}), 4.44(\mathrm{~s}, 2 \mathrm{H}), 3.80(\mathrm{~s}, 3 \mathrm{H}), 3.72(\mathrm{~s}, 3 \mathrm{H}), 3.67$ (s, $6 \mathrm{H}), 2.32(\mathrm{~d}, J=0.9 \mathrm{~Hz}, 3 \mathrm{H}) ;{ }^{13} \mathrm{C} \mathrm{NMR}\left(100 \mathrm{MHz}, \mathrm{CDCl}_{3}\right)$ $\delta: 165.3,160.1,160.1,158.3,154.0,152.8,151.5,137.3$, $134.3,129.7,128.0,124.6,113.0,112.8,112.2$, 111.2, $104.4,100.2,65.1,60.0,55.3,54.3,51.7,17.7$; HRMS calcd for $\mathrm{C}_{29} \mathrm{H}_{30} \mathrm{~N}_{2} \mathrm{O}_{7}[\mathrm{M}+\mathrm{H}]^{+}$520.1966, found 520.1963.

$N$-(4-甲基苄基)-2-((4-甲基- $2 H$-色烯-2-酮-7-基)氧 基)- $N-(3,4,5-$ 三甲氧基苯基)乙酰胺(4c): 白色固体, 收率 63\%. m.p. 166 167 ${ }^{\circ} \mathrm{C}$; ${ }^{1} \mathrm{H}$ NMR (400 MHz, $\left.\mathrm{CDCl}_{3}\right) \delta$ : 7.42 (d, $J=8.8 \mathrm{~Hz}, 1 \mathrm{H}), 7.04$ (t, $J=5.6 \mathrm{~Hz}, 4 \mathrm{H}), 6.82$ (dd, $J=8.8,2.5 \mathrm{~Hz}, 1 \mathrm{H}), 6.58(\mathrm{~d}, J=2.4 \mathrm{~Hz}, 1 \mathrm{H}), 6.12(\mathrm{~s}, 2 \mathrm{H})$, $6.06(\mathrm{~d}, J=1.0 \mathrm{~Hz}, 1 \mathrm{H}), 4.75$ (s, 2H), 4.45 (s, 2H), 3.79 (s, $3 \mathrm{H}), 3.66$ (s, 6H), 2.32 (d, $J=0.9 \mathrm{~Hz}, 3 \mathrm{H}), 2.25$ (s, 3H); ${ }^{13} \mathrm{C}$ NMR (100 MHz, $\left.\mathrm{CDCl}_{3}\right) \delta: 167.1,132.9,130.2,129.5$, 128.3, 96.39, 95.3, 94.8, 93.2, 92.3, 91.9, 91.9, 77.4, 77.1, 76.8, 55.2, 52.1, 44.8, 29.5, 27.1; HRMS calcd for
$\mathrm{C}_{29} \mathrm{H}_{29} \mathrm{NO}_{7} \mathrm{Na}[\mathrm{M}+\mathrm{Na}]^{+}$526.1836, found 526.1841.

$N$-(4-氟茮基)-2-((4-甲基-2 $H$-色烯-2-酮-7-基)氧 基)- $N$-(3,4,5-三甲氧基苯基)乙酰胺(4d)：白色固体，收 率 86\%. m.p. $131 \sim 132{ }^{\circ} \mathrm{C} ;{ }^{1} \mathrm{H}$ NMR (400 $\mathrm{MHz}, \mathrm{CDCl}_{3}$ ) $\delta: 7.42(\mathrm{~d}, J=8.8 \mathrm{~Hz}, 1 \mathrm{H}), 7.15(\mathrm{dd}, J=8.5,5.4 \mathrm{~Hz}, 2 \mathrm{H})$, $6.91(\mathrm{t}, J=8.6 \mathrm{~Hz}, 2 \mathrm{H}), 6.82(\mathrm{dd}, J=8.8,2.4 \mathrm{~Hz}, 1 \mathrm{H}), 6.58$ $(\mathrm{d}, J=2.4 \mathrm{~Hz}, 1 \mathrm{H}), 6.12(\mathrm{~s}, 2 \mathrm{H}), 6.07$ (d, $J=0.9 \mathrm{~Hz}, 1 \mathrm{H})$, $4.76(\mathrm{~s}, 2 \mathrm{H}), 4.45$ (s, 2H), $3.80(\mathrm{~s}, 3 \mathrm{H}), 3.68(\mathrm{~s}, 6 \mathrm{H}), 2.32$ $(\mathrm{d}, J=0.9 \mathrm{~Hz}, 3 \mathrm{H}) ;{ }^{13} \mathrm{C} \mathrm{NMR}\left(100 \mathrm{MHz}, \mathrm{CDCl}_{3}\right) \delta: 165.5$, $160.1,159.9$, 154.0, 152.9, 151.5, 137.7, 134.2, 131.8, $131.7,130.2,130.1,124.7,114.5,114.3,113.1,112.1$, 111.2, 104.3, 100.2, 65.0, 60.0, 55.3, 51.6, 17.6; HRMS calcd for $\mathrm{C}_{28} \mathrm{H}_{26} \mathrm{FNO}_{7} \mathrm{Na}[\mathrm{M}+\mathrm{Na}]^{+}$530.1586, found 530.1592

$N$-(4-溴苄基)-2-((4-甲基- $2 H$-色烯-2-酮-7-基)氧 基)- $N$-(3,4,5-三甲氧基苯基)乙酰胺(4e)：白色固体，收率 65\%. m.p. 181 $182{ }^{\circ} \mathrm{C} ;{ }^{1} \mathrm{H}$ NMR (400 MHz, $\left.\mathrm{CDCl}_{3}\right) \delta$ : 7.49 (d, $J=8.8 \mathrm{~Hz}, 1 \mathrm{H}), 7.43$ (d, $J=8.3 \mathrm{~Hz}, 2 \mathrm{H}), 7.13$ (d, $J=8.3 \mathrm{~Hz}, 2 \mathrm{H}), 6.89(\mathrm{dd}, J=8.8,2.5 \mathrm{~Hz}, 1 \mathrm{H}), 6.65$ (d, $J=$ $2.5 \mathrm{~Hz}, 1 \mathrm{H}), 6.20(\mathrm{~s}, 2 \mathrm{H}), 6.14(\mathrm{~d}, J=1.1 \mathrm{~Hz}, 1 \mathrm{H}), 4.81(\mathrm{~s}$, 2H), 4.53 (s, 2H), 3.87 (s, 3H), $3.76(\mathrm{~s}, 6 \mathrm{H}), 2.39$ (d, $J=$ $1.0 \mathrm{~Hz}, 3 \mathrm{H}) ;{ }^{13} \mathrm{C} \mathrm{NMR}\left(100 \mathrm{MHz}, \mathrm{CDCl}_{3}\right) \delta: 165.6,160.1$, $159.9,153.9,152.9,151.5,137.5,134.8,134.2,130.6$, $130.1,124.7,120.9,113.1,112.1,111.2,104.3,100.2$, $64.9,60.0,55.3,51.8,17.7$; HRMS calcd for $\mathrm{C}_{28} \mathrm{H}_{26} \mathrm{BrN}-$ $\mathrm{O}_{7} \mathrm{Na}[\mathrm{M}+\mathrm{Na}]^{+}$590.0785, found 590.0790 .

$N$-(4-氯芳基)-2-((4-甲基- $2 H$-色烯-2-酮-7-基)氧 基)- $N$-(3,4,5-三甲氧基苯基)乙酰胺(4f)：白色固体，收率 74\%. m.p. 155 156 ${ }^{\circ} \mathrm{C} ;{ }^{1} \mathrm{H}$ NMR (400 MHz, $\left.\mathrm{CDCl}_{3}\right) \delta$ : 7.42 (d, $J=8.8 \mathrm{~Hz}, 1 \mathrm{H}), 7.21$ (d, $J=4.2 \mathrm{~Hz}, 1 \mathrm{H}), 7.19$ (s, $1 \mathrm{H}), 7.11(\mathrm{~d}, J=8.4 \mathrm{~Hz}, 2 \mathrm{H}), 6.81(\mathrm{dd}, J=8.8,2.3 \mathrm{~Hz}$, $1 \mathrm{H}), 6.58$ (d, $J=2.3 \mathrm{~Hz}, 1 \mathrm{H}), 6.13$ (s, 2H), 6.07 (d, $J=0.8$ $\mathrm{Hz}, 1 \mathrm{H}), 4.76$ (s, 2H), 4.46 (s, 2H), 3.80 (s, 3H), 3.69 (s, $6 \mathrm{H}), 2.32(\mathrm{~d}, J=0.7 \mathrm{~Hz}, 3 \mathrm{H}) ;{ }^{13} \mathrm{C} \mathrm{NMR}\left(100 \mathrm{MHz}, \mathrm{CDCl}_{3}\right)$ $\delta: 165.6,160.1,159.9,154.0,153.0,151.5,137.5,134.4$, $134.2,132.8,129.8,127.7,124.7,113.1,112.1,111.3$, 104.3, 100.2, 65.0, 60.0, 55.3, 51.7, 17.7; HRMS calcd for $\mathrm{C}_{28} \mathrm{H}_{27} \mathrm{ClNO}_{7}[\mathrm{M}+\mathrm{H}]^{+}$524.1471, found 524.1477.

$\mathrm{N}$-(3-甲氧基苠基)-2-((4-甲基- $2 H$-色烯-2-酮-7-基)氧 基)- $N$-(3,4,5-三甲氧基苯基)乙酰胺(4g)：白色固体，收 率 82\%. m.p. $131 \sim 132{ }^{\circ} \mathrm{C} ;{ }^{1} \mathrm{H}$ NMR (400 MHz, $\mathrm{CDCl}_{3}$ ) $\delta: 7.42(\mathrm{~d}, J=8.8 \mathrm{~Hz}, 1 \mathrm{H}), 7.13(\mathrm{t}, J=8.0 \mathrm{~Hz}, 1 \mathrm{H}), 6.82$ (dd, $J=8.8,2.5 \mathrm{~Hz}, 1 \mathrm{H}), 6.74(\mathrm{dd}, J=12.8,4.0 \mathrm{~Hz}, 3 \mathrm{H})$, $6.59(\mathrm{~d}, J=2.4 \mathrm{~Hz}, 1 \mathrm{H}), 6.15(\mathrm{~s}, 2 \mathrm{H}), 6.06(\mathrm{~s}, 1 \mathrm{H}), 4.76(\mathrm{~s}$, 2H), 4.47 (s, 2H), 3.79 (s, 3H), 3.67 (d, $J=1.3 \mathrm{~Hz}, 9 \mathrm{H})$, 
$2.32(\mathrm{~s}, 3 \mathrm{H}) ;{ }^{13} \mathrm{C} \mathrm{NMR}\left(100 \mathrm{MHz}, \mathrm{CDCl}_{3}\right) \delta: 165.4,160.1$, $160.0,158.7,154.0,152.9,151.5,137.4,134.4,128.5$, $124.6,120.6,113.8,113.1,112.4,112.1,111.2,104.4$, 100.2, 65.1, 60.0, 55.3, 54.3, 52.3, 17.6; HRMS calcd for $\mathrm{C}_{29} \mathrm{H}_{30} \mathrm{NO}_{8}[\mathrm{M}+\mathrm{H}]^{+}$520.1966, found 520.1985.

$N$-(3-甲基苄基)-2-((4-甲基- $2 H$-色烯-2-酮-7-基)氧 基)- $N$-(3,4,5-三甲氧基苯基)乙酰胺(4h): 白色固体, 收 率 82\%. m.p. $149 \sim 150{ }^{\circ} \mathrm{C} ;{ }^{1} \mathrm{H}$ NMR (400 $\mathrm{MHz}, \mathrm{CDCl}_{3}$ ) $\delta: 7.42(\mathrm{~d}, J=8.8 \mathrm{~Hz}, 1 \mathrm{H}), 7.10(\mathrm{t}, J=7.5 \mathrm{~Hz}, 1 \mathrm{H}), 7.04 \sim$ $6.95(\mathrm{~m}, 2 \mathrm{H}), 6.93(\mathrm{~d}, J=7.4 \mathrm{~Hz}, 1 \mathrm{H}), 6.83(\mathrm{dd}$, $J=8.8,2.5 \mathrm{~Hz}, 1 \mathrm{H}), 6.59$ (d, $J=2.4 \mathrm{~Hz}, 1 \mathrm{H}), 6.13(\mathrm{~s}, 2 \mathrm{H})$, $6.06(\mathrm{~d}, J=1.0 \mathrm{~Hz}, 1 \mathrm{H}), 4.75(\mathrm{~s}, 2 \mathrm{H}), 4.46$ (s, 2H), 3.79 (s, $3 \mathrm{H}), 3.66(\mathrm{~s}, 6 \mathrm{H}), 2.31(\mathrm{~d}, J=0.9 \mathrm{~Hz}, 3 \mathrm{H}), 2.22$ (s, 3H); ${ }^{13} \mathrm{C}$ NMR (100 MHz, $\left.\mathrm{CDCl}_{3}\right) \delta: 165.4,160.1,154.0,152.8$, $151.5,137.4,137.2,135.8,134.4,129.0,127.5,127.4$, $125.4,124.63,113.1,112.1,111.2,104.4,100.2,65.1$, $60.0,55.2,52.3,20.3,17.6$; HRMS calcd for $\mathrm{C}_{29} \mathrm{H}_{29} \mathrm{NO}_{7} \mathrm{Na}$ $[\mathrm{M}+\mathrm{Na}]^{+}$526.1842, found 526.1843.

$N$-(3-氟苄基)-2-((4-甲基- $2 H$-色烯-2-酮-7-基)氧 基)- $N-(3,4,5$-三甲氧基苯基)乙酰胺(4i): 白色固体, 收率 62\%. m.p. 143 $144{ }^{\circ} \mathrm{C} ;{ }^{1} \mathrm{H}$ NMR (400 MHz, $\left.\mathrm{CDCl}_{3}\right) \delta$ : 7.42 (d, $J=8.8 \mathrm{~Hz}, 1 \mathrm{H}), 7.19$ (t, $J=11.2 \mathrm{~Hz}, 1 \mathrm{H}), 6.95$ (d, $J=7.9 \mathrm{~Hz}, 1 \mathrm{H}), 6.93 \sim 6.88(\mathrm{~m}, 2 \mathrm{H}), 6.82(\mathrm{dd}, J=8.8$, $2.4 \mathrm{~Hz}, 1 \mathrm{H}), 6.58(\mathrm{~d}, J=2.3 \mathrm{~Hz}, 1 \mathrm{H}), 6.15(\mathrm{~s}, 2 \mathrm{H}), 6.06(\mathrm{~s}$, $1 \mathrm{H}), 4.78(\mathrm{~s}, 2 \mathrm{H}), 4.47(\mathrm{~s}, 2 \mathrm{H}), 3.80(\mathrm{~s}, 3 \mathrm{H}), 3.68(\mathrm{~s}, 6 \mathrm{H})$, $2.32(\mathrm{~s}, 3 \mathrm{H}) ;{ }^{13} \mathrm{C}$ NMR $\left(100 \mathrm{MHz}, \mathrm{CDCl}_{3}\right) \delta: 165.6,163.0$, $160.5,160.0,159.9,154.0,153.0,151.5,138.4,138.3$, $137.5,134.3,129.1,129.0,124.7,123.9,123.9,115.3$, $115.0,113.9,113.7,113.2,112.1,111.3,104.3,100.3,65.0$, 60.0, 55.3, 51.9, 17.6; HRMS calcd for $\mathrm{C}_{28} \mathrm{H}_{26} \mathrm{FNO}_{7} \mathrm{Na}$ $[\mathrm{M}+\mathrm{Na}]^{+}$530.1591, found 530.1592.

$N$-(3-氯苄基)-2-((4-甲基- $2 H$-色烯-2-酮-7-基)氧 基)- $N$-(3,4,5-三甲氧基苯基)乙酰胺 $(\mathbf{4} \mathbf{j})$ : 白色固体, 收率 64\%. m.p. 145 146 ${ }^{\circ} \mathrm{C} ;{ }^{1} \mathrm{H}$ NMR (400 MHz, $\left.\mathrm{CDCl}_{3}\right) \delta$ : $7.42(\mathrm{~d}, J=8.8 \mathrm{~Hz}, 1 \mathrm{H}), 7.22 \sim 7.12(\mathrm{~m}, 3 \mathrm{H}), 7.08(\mathrm{~d}, J=$ $7.2 \mathrm{~Hz}, 1 \mathrm{H}), 6.81(\mathrm{dd}, J=8.8,2.5 \mathrm{~Hz}, 1 \mathrm{H}), 6.58$ (d, $J=2.4$ $\mathrm{Hz}, 1 \mathrm{H}), 6.15$ (s, 2H), 6.06 (d, J=1.0 Hz, 1H), 4.76 (s, $2 \mathrm{H}), 4.47$ (s, 2H), $3.80(\mathrm{~s}, 3 \mathrm{H}), 3.69(\mathrm{~s}, 6 \mathrm{H}), 2.31(\mathrm{~d}, J=$ $1.0 \mathrm{~Hz}, 3 \mathrm{H}) ;{ }^{13} \mathrm{C} \mathrm{NMR}\left(100 \mathrm{MHz}, \mathrm{CDCl}_{3}\right) \delta: 165.6,160.0$, $159.9,154.0,153.0,151.5,137.9,137.6,134.2,133.3$, $128.9,128.3,127.1,126.5,124.7,113.1,112.0,111.3$, 104.3, 100.3, 65.0, 60.0, 55.3, 51.8, 17.6; HRMS calcd for $\mathrm{C}_{28} \mathrm{H}_{26} \mathrm{ClNO}_{7} \mathrm{Na}[\mathrm{M}+\mathrm{Na}]^{+}$546.1290, found 546.1295.

$N$-((1H-苯并 $[d]$ 咪唑-2-基)甲基)-2-((4-甲基- $2 H$-色 烯-2-酮-7-基)氧基)- $N$-(3,4,5-三甲氧基苯基)乙酰胺(4k):
白色固体，收率 60\%. m.p. 146 $147{ }^{\circ} \mathrm{C} ;{ }^{1} \mathrm{H}$ NMR (400 $\left.\mathrm{MHz} \mathrm{CDCl}_{3}\right) \delta: 7.36(\mathrm{~d}, J=8.8 \mathrm{~Hz}, 1 \mathrm{H}), 7.18$ (dd, $J=5.8$, $3.1 \mathrm{~Hz}, 2 \mathrm{H}), 6.69$ (t, $J=18.4 \mathrm{~Hz}, 1 \mathrm{H}), 6.61(\mathrm{~s}, 1 \mathrm{H}), 6.34$ (s, 2H), 6.06 (s, 1H), 4.98 (s, 2H), 4.54 (s, 2H), 3.79 (s, 3H), $3.70(\mathrm{~s}, 6 \mathrm{H}), 2.29(\mathrm{~s}, 3 \mathrm{H}) ;{ }^{13} \mathrm{C} \mathrm{NMR}\left(101 \mathrm{MHz}, \mathrm{CDCl}_{3}\right) \delta$ : $167.7,159.9,159.7,154.0,153.3,151.3,148.7,138.0$, $134.5,124.7,121.9,113.3,111.6,111.5,103.8,100.5$, 64.9, 59.9, 55.4, 47.8, 17.6; HRMS calcd for $\mathrm{C}_{29} \mathrm{H}_{28} \mathrm{~N}_{3} \mathrm{O}_{7}$ $[\mathrm{M}+\mathrm{H}]^{+}$530.1922, found 530.1923.

$N$-((苯并 $[d]$ 噻唑-2-基)甲基)-2-((4-甲基- $2 H$-色烯-2酮-7-基)氧基)- $N-(3,4,5$-三甲氧基苯基)乙酰胺(41): 白色 固体, 收率 62\%. m.p. 175 $176{ }^{\circ} \mathrm{C} ;{ }^{1} \mathrm{H}$ NMR (400 MHz, $\left.\mathrm{CDCl}_{3}\right) \delta: 7.94(\mathrm{~d}, J=8.1 \mathrm{~Hz}, 1 \mathrm{H}), 7.82(\mathrm{~d}, J=7.9 \mathrm{~Hz}$, 1H), 7.40 (d, $J=8.8 \mathrm{~Hz}, 2 \mathrm{H}), 7.33$ (t, $J=7.5 \mathrm{~Hz}, 1 \mathrm{H}), 6.82$ (dd, $J=8.8,2.4 \mathrm{~Hz}, 1 \mathrm{H}), 6.66(\mathrm{~d}, J=2.4 \mathrm{~Hz}, 1 \mathrm{H}), 6.50$ (s, $2 \mathrm{H}), 6.06(\mathrm{~s}, 1 \mathrm{H}), 5.21(\mathrm{~s}, 2 \mathrm{H}), 4.58(\mathrm{~s}, 2 \mathrm{H}), 3.79(\mathrm{~s}, 3 \mathrm{H})$, $3.71(\mathrm{~s}, 6 \mathrm{H}), 2.31(\mathrm{~s}, 3 \mathrm{H}) ;{ }^{13} \mathrm{C} \mathrm{NMR}\left(100 \mathrm{MHz}, \mathrm{CDCl}_{3}\right) \delta$ : $166.1,165.5,160.0,159.9,154.0,153.2,151.4,137.8$, $134.6,134.4,125.3,124.7,124.5,122.1,120.8,113.2$, $112.1,111.3,104.1,100.4,65.0,60.0,59.4,55.3,50.8$, 20.0, 17.6, 13.2; HRMS calcd for $\mathrm{C}_{29} \mathrm{H}_{27} \mathrm{~N}_{2} \mathrm{O}_{7} \mathrm{~S}[\mathrm{M}+\mathrm{H}]^{+}$ 547.1533 , found 547.1538 .

$\mathrm{N}$-(吡啶-2-基甲基)-2-((4-甲基- $2 \mathrm{H}$-色烯-2-酮-7-基) 氧基)- $N$-(3,4,5-三甲氧基苯基)乙酰胺(4m): 白色固体, 收率 55\%. m.p. $142 \sim 143{ }^{\circ} \mathrm{C} ;{ }^{1} \mathrm{H}$ NMR (400 MHz, $\left.\mathrm{CDCl}_{3}\right) \delta: 8.54(\mathrm{~d}, J=4.4 \mathrm{~Hz}, 1 \mathrm{H}), 7.60(\mathrm{td}, J=7.7,1.5$ $\mathrm{Hz}, 1 \mathrm{H}), 7.40$ (d, $J=8.8 \mathrm{~Hz}, 1 \mathrm{H}), 7.31$ (d, $J=7.8 \mathrm{~Hz}, 1 \mathrm{H})$, $7.17 \sim 7.08(\mathrm{~m}, 1 \mathrm{H}), 6.82(\mathrm{dd}, J=8.8,2.4 \mathrm{~Hz}, 1 \mathrm{H}), 6.67(\mathrm{~d}$, $J=2.3 \mathrm{~Hz}, 1 \mathrm{H}), 6.46(\mathrm{~s}, 2 \mathrm{H}), 6.06(\mathrm{~s}, 1 \mathrm{H}), 4.95(\mathrm{~s}, 2 \mathrm{H})$, 4.55 (s, 2H), 3.78 (s, 3H), $3.72(\mathrm{~s}, 6 \mathrm{H}), 2.31(\mathrm{~s}, 3 \mathrm{H}) ;{ }^{13} \mathrm{C}$ NMR (100 MHz, $\left.\mathrm{CDCl}_{3}\right) \delta: 166.0,160.1,155.3,154.0$, $152.9,151.5,148.1,137.4,136.0,135.2,124.6,122.1$, $121.7,113.0,112.2,111.1,104.3,100.4,65.1,59.9,55.3$, 54.1, 17.6; HRMS calcd for $\mathrm{C}_{30} \mathrm{H}_{27} \mathrm{ClNO}_{7} \mathrm{~S}[\mathrm{M}+\mathrm{H}]^{+}$ 491.1813, found 491.1817 .

$N$-((5-氯苯并 $[b]$ 噻吩-3-基)甲基)-2-((4-甲基- $2 H$ 色烯-2-酮-7-基)氧基)- $N$-(3,4,5-三甲氧基苯基) 乙酰胺 (4n): 白色固体, 收率 53\%. m.p. 172 174 ${ }^{\circ} \mathrm{C} ;{ }^{1} \mathrm{H}$ NMR $\left(400 \mathrm{MHz}, \mathrm{CDCl}_{3}\right) \delta: 7.69(\mathrm{~d}, J=8.6 \mathrm{~Hz}, 1 \mathrm{H}), 7.61(\mathrm{~d}, J=$ $1.9 \mathrm{~Hz}, 1 \mathrm{H}), 7.43$ (d, $J=8.8 \mathrm{~Hz}, 1 \mathrm{H}), 7.23$ (dd, $J=8.6,1.9$ $\mathrm{Hz}, 1 \mathrm{H}), 6.82(\mathrm{dd}, J=8.8,2.5 \mathrm{~Hz}, 1 \mathrm{H}), 6.53(\mathrm{~d}, J=2.5 \mathrm{~Hz}$, $1 \mathrm{H}), 6.06(\mathrm{~s}, 1 \mathrm{H}), 6.05(\mathrm{~s}, 2 \mathrm{H}), 5.02(\mathrm{~s}, 2 \mathrm{H}), 4.45(\mathrm{~s}, 2 \mathrm{H})$, $3.79(\mathrm{~s}, 3 \mathrm{H}), 3.60(\mathrm{~s}, 6 \mathrm{H}), 2.32(\mathrm{~s}, 3 \mathrm{H}) ;{ }^{13} \mathrm{C}$ NMR $(100$ $\left.\mathrm{MHz}, \mathrm{CDCl}_{3}\right) \delta: 165.6,160.0,159.9,154.0,153.0,151.4$, $138.3,137.4,133.8,130.1,130.0,128.3,124.7,124.1$, 
$122.8,121.1,113.2,111.9,111.3,104.6,100.3,65.1,60.0$, 55.3, 45.1, 17.6; HRMS calcd for $\mathrm{C}_{30} \mathrm{H}_{26} \mathrm{ClNO}_{7} \mathrm{SNa}[\mathrm{M}+$ $\mathrm{Na}]^{+}$602.1011, found 602.1016.

$N$-((5-氯苯并 $[b]$ 噻吩-3-基)甲基)-2-((2H-色烯-2酮-7-基)氧基)- $N$-(3,4,5-三甲氧基苯基)乙酰胺(4o): 白色 固体, 收率 87\%. m.p. 179 $180{ }^{\circ} \mathrm{C} ;{ }^{1} \mathrm{H}$ NMR $(400 \mathrm{MHz}$, $\left.\mathrm{CDCl}_{3}\right) \delta: 7.69(\mathrm{~d}, J=8.6 \mathrm{~Hz}, 1 \mathrm{H}), 7.61(\mathrm{~d}, J=1.7 \mathrm{~Hz}$, $1 \mathrm{H}), 7.56$ (d, $J=9.5 \mathrm{~Hz}, 1 \mathrm{H}), 7.30$ (d, $J=8.5 \mathrm{~Hz}, 1 \mathrm{H}), 7.23$ (dd, $J=8.6,1.8 \mathrm{~Hz}, 1 \mathrm{H}), 6.80$ (d, $J=6.7 \mathrm{~Hz}, 1 \mathrm{H}), 6.54$ (s, $1 \mathrm{H}), 6.18(\mathrm{~d}, J=9.5 \mathrm{~Hz}, 1 \mathrm{H}), 6.05(\mathrm{~s}, 2 \mathrm{H}), 5.02(\mathrm{~s}, 2 \mathrm{H})$, 4.45 (s, 2H), 3.79 (s, 3H), $3.59(\mathrm{~s}, 6 \mathrm{H}) ;{ }^{13} \mathrm{C}$ NMR (100 $\left.\mathrm{MHz} \mathrm{CDCl}_{3}\right) \delta: 160.1,159.9,154.6,153.0,142.3,138.2$, $137.7,137.3,133.7,130.0,129.9,128.3,127.9$, 124.1, 122.8, 121.0, 112.5, 112.3, 112.1, 104.5, 100.2, 65.1, 60.0, 55.3, 45.1; HRMS calcd for $\mathrm{C}_{29} \mathrm{H}_{24} \mathrm{ClNO}_{7} \mathrm{SNa}[\mathrm{M}+\mathrm{Na}]^{+}$ 588.0854, found 588.0860.

$N$-((5-氯苯并 $[b]$ 噻吩-3-基)甲基)-2-((3-氯-4-甲基$2 H$-色烯-2-酮-7-基)氧基)- $N$-(3,4,5-三甲氧基苯基)乙酰 胺(4p): 白色固体, 收率 36\%. m.p. $188 \sim 189{ }^{\circ} \mathrm{C} ;{ }^{1} \mathrm{H}$ NMR (400 MHz, $\left.\mathrm{CDCl}_{3}\right) \delta: 7.69(\mathrm{~d}, J=8.6 \mathrm{~Hz}, 1 \mathrm{H}), 7.60$ $(\mathrm{d}, J=1.8 \mathrm{~Hz}, 1 \mathrm{H}), 7.45(\mathrm{~d}, J=8.9 \mathrm{~Hz}, 1 \mathrm{H}), 7.22(\mathrm{dd}, J=$ 8.6, $1.8 \mathrm{~Hz}, 1 \mathrm{H}), 7.18$ (s, 1H), 6.86 (dd, $J=8.9,2.5 \mathrm{~Hz}$, $1 \mathrm{H}), 6.53(\mathrm{~d}, J=2.4 \mathrm{~Hz}, 1 \mathrm{H}), 6.04$ (s, 2H), 5.01 (s, 2H), $4.45(\mathrm{~s}, 2 \mathrm{H}), 3.79(\mathrm{~s}, 3 \mathrm{H}), 3.60(\mathrm{~s}, 6 \mathrm{H}), 2.47(\mathrm{~s}, 3 \mathrm{H}) ;{ }^{13} \mathrm{C}$ NMR $\left(100 \mathrm{MHz}, \mathrm{CDCl}_{3}\right) \delta: 165.4,159.8,156.1,153.0$, $151.8,146.8,138.2,137.7,137.4,133.7,130.0,129.9$, $128.3,125.1,124.1,122.8,121.0,117.1,112.8,112.6$, $104.5,100.1,65.0,60.0,55.3,45.0,15.2$; HRMS calcd for $\mathrm{C}_{30} \mathrm{H}_{26} \mathrm{Cl}_{2} \mathrm{NO}_{7} \mathrm{~S}[\mathrm{M}+\mathrm{H}]^{+}$614.0802, found 614.0815.

$N$-((5-氯苯并 $[b]$ 噻吩-3-基)甲基)-2-((2H-色烯-2酮-4-基)氧基)- $N$-(3,4,5-三甲氧基苯基)乙酰胺(4q): 白色 固体, 收率 68\%. m.p. 223 224 ${ }^{\circ} \mathrm{C} ;{ }^{1} \mathrm{H}$ NMR $(400 \mathrm{MHz}$, $\left.\mathrm{CDCl}_{3}\right) \delta: 7.80(\mathrm{dd}, J=7.9,1.2 \mathrm{~Hz}, 1 \mathrm{H}), 7.68(\mathrm{~d}, J=8.5$ $\mathrm{Hz}, 1 \mathrm{H}), 7.59$ (d, $J=1.8 \mathrm{~Hz}, 1 \mathrm{H}), 7.54 \sim 7.42(\mathrm{~m}, 1 \mathrm{H})$, $7.26 \sim 7.24$ (m, 1H), 7.23 (s, 2H), 7.20 (d, $J=7.2 \mathrm{~Hz}, 1 \mathrm{H})$, $6.05(\mathrm{~s}, 2 \mathrm{H}), 5.39(\mathrm{~s}, 1 \mathrm{H}), 5.03(\mathrm{~s}, 2 \mathrm{H}), 4.55(\mathrm{~s}, 2 \mathrm{H}), 3.79$ $(\mathrm{s}, 3 \mathrm{H}), 3.56(\mathrm{~s}, 6 \mathrm{H}) ;{ }^{13} \mathrm{C} \mathrm{NMR}\left(100 \mathrm{MHz}, \mathrm{CDCl}_{3}\right) \delta$ : $163.9,161.5,153.1,152.4,138.3,137.9,137.3,133.5$, $131.6,130.0,129.8,128.4,124.1,123.0,122.8,122.3$, $121.0,115.7,114.3,104.5,90.1,65.2,60.0,59.4,55.2$, 45.2; HRMS calcd for $\mathrm{C}_{29} \mathrm{H}_{25} \mathrm{ClNO}_{7} \mathrm{~S}[\mathrm{M}+\mathrm{H}]^{+}$566.1035, found 566.1026 .

$N$-((5-氯苯并 $[b]$ 噻吩-3-基)甲基)-2-((4-甲基- $2 H$-色 烯-2-酮-6-基)氧基)- $N$-(3,4,5-三甲氧基苯基)乙酰胺(4r): 白色固体，收率 73\%. m.p. 169 170 ${ }^{\circ} \mathrm{C} ;{ }^{1} \mathrm{H}$ NMR (400
$\left.\mathrm{MHz}, \mathrm{CDCl}_{3}\right) \delta: 7.69(\mathrm{~d}, J=8.6 \mathrm{~Hz}, 1 \mathrm{H}), 7.63(\mathrm{~d}, J=1.7$ $\mathrm{Hz}, 1 \mathrm{H}), 7.24$ (dd, $J=8.6,1.8 \mathrm{~Hz}, 1 \mathrm{H}), 7.16(\mathrm{~d}, J=8.7 \mathrm{~Hz}$, $1 \mathrm{H}), 6.92(\mathrm{~s}, 1 \mathrm{H}), 6.90(\mathrm{~d}, J=2.9 \mathrm{~Hz}, 1 \mathrm{H}), 6.20(\mathrm{~s}, 1 \mathrm{H})$, $6.01(\mathrm{~s}, 2 \mathrm{H}), 5.02(\mathrm{~s}, 2 \mathrm{H}), 4.44(\mathrm{~s}, 2 \mathrm{H}), 3.78(\mathrm{~s}, 3 \mathrm{H}), 3.56$ $(\mathrm{s}, 6 \mathrm{H}), 2.19(\mathrm{~d}, J=0.7 \mathrm{~Hz}, 3 \mathrm{H}) ;{ }^{13} \mathrm{C}$ NMR $(100 \mathrm{MHz}$, $\mathrm{CDCl}_{3}$ ) $\delta: 166.1,159.8,153.3,152.9,150.7,147.3,138.3$, $137.7,137.3,134.0,130.2,130.0,128.2,124.2,122.8$, $121.1,119.5,117.5,116.9,114.6,108.8,104.6,65.8,60.0$, $55.2,45.1,17.5$; HRMS calcd for $\mathrm{C}_{30} \mathrm{H}_{27} \mathrm{ClNO}_{7} \mathrm{~S}[\mathrm{M}+\mathrm{H}]^{+}$ 580.1191 , found 580.1097 .

$N$-((5-氯苯并 $[b]$ 噻吩-3-基)甲基)-2-((7-甲基- $2 H$ 色烯-2-酮-4-基)氧基)- $N$-(3,4,5-三甲氧基苯基)乙酰胺 (4s): 白色固体，收率 59\%. m.p. 219 220 ${ }^{\circ} \mathrm{C} ;{ }^{1} \mathrm{H}$ NMR $\left(400 \mathrm{MHz}, \mathrm{CDCl}_{3}\right) \delta: 7.68(\mathrm{dd}, J=8.3,5.2 \mathrm{~Hz}, 2 \mathrm{H}), 7.59$ $(\mathrm{d}, J=1.7 \mathrm{~Hz}, 1 \mathrm{H}), 7.27 \sim 7.24(\mathrm{~m}, 1 \mathrm{H}), 7.23(\mathrm{~s}, 1 \mathrm{H})$, $7.08 \sim 6.98(\mathrm{~m}, 2 \mathrm{H}), 6.04(\mathrm{~s}, 2 \mathrm{H}), 5.34(\mathrm{~d}, J=16.6 \mathrm{~Hz}$, $1 \mathrm{H}), 5.03(\mathrm{~s}, 2 \mathrm{H}), 4.53(\mathrm{~s}, 2 \mathrm{H}), 3.79(\mathrm{~s}, 3 \mathrm{H}), 3.55(\mathrm{~s}, 6 \mathrm{H})$, 2.37 (s, 3H); ${ }^{13} \mathrm{C}$ NMR (100 MHz, $\left.\mathrm{CDCl}_{3}\right) \delta: 164.2,164.0$, $161.8,153.1,152.5,142.9,138.3,137.8,137.3,133.5$, $130.0,129.8,128.4,124.2,124.1,122.8,122.0,121.0$, $115.8,111.8,104.4,89.2,65.2,60.0,59.4,55.2,45.2,20.7$; HRMS calcd for $\mathrm{C}_{30} \mathrm{H}_{27} \mathrm{ClNO}_{7} \mathrm{~S}[\mathrm{M}+\mathrm{H}]^{+}$580.1191, found 580.1201 .

\section{3 抗肿瘤活性测试}

测试所用的肿瘤细胞由郑州大学基础医学院药理 系提供. 所有化合物均用二甲基亚砜(DMSO)溶解，配 制成初始浓度为 $20 \mathrm{mmol} / \mathrm{L}$ 的储备液, 放于 $4{ }^{\circ} \mathrm{C}$ 冰箱中 保存，使用前用培养基稀释成所需浓度，使 DMSO 的终 含量不超过 $0.1 \%$. 将肿瘤细胞培养于含 $5 \% \mathrm{CO}_{2}$ 的 RPMI-1640 培养基中, 加入适量的 $10 \%$ 胎牛血清以及 $100 \mathrm{U} / \mathrm{mL}$ 的青霉素和 $0.1 \mathrm{mg} / \mathrm{mL}$ 的链霉素, 摇晃均匀, 配成细胞悬液. 将细胞悬液按每孔 $100 \mu \mathrm{L}$ 于 96 孔板中 培养 $24 \mathrm{~h}$ 后, 弃去培养基, 然后加入不同浓度的药物, 每孔 $200 \mu \mathrm{L}$. 培养 $48 \mathrm{~h}$ 后, 取出放在超净台中操作，每 孔加入 $5 \mathrm{~g} / \mathrm{L}$ 的四甲基偶氮唑盐(MTT)溶液 $20 \mu \mathrm{L}$, 继续 置于培养箱中培养 $4 \mathrm{~h}$ 后, 取出至实验台, 移除 96 孔板 中培养基，每孔加入 $100 \mu \mathrm{L}$ 二甲基亚砜(DMSO)溶解的 蓝紫色甲瑎颗粒，置于摇床摇 $10 \mathrm{~min}$ 后，用酶标仪测定 $570 \mathrm{~nm}$ 处的吸光度值 $\mathrm{A}$, 对所得数值进行处理, 分别计 算出 5-氟尿嘧啶和各化合物的平均值、标准差和存活率. 通过存活率柱状图观察不同浓度药物作用的梯度情况， 用 SPSS 软件计算每个化合物的 $\mathrm{IC}_{50}$.

辅助材料(Supporting Information) 化合物 $\mathbf{2 a} \sim \mathbf{2 n}$ 、 $3 a \sim 3 n$ 和 $4 a \sim 4 s$ 的 ${ }^{1} \mathrm{H} \mathrm{NMR}$ 和 ${ }^{13} \mathrm{C} \mathrm{NMR}$ 谱图. 这些材 
料可以免费从本刊网站(http://sioc-journal.cn/)上下载.

\section{References}

[1] Zhang, S.-Y.; Meng, L.; Gao, W.-Y.; Song, N.-Y.; Jia, W.; Duan, H.-Q. China J. Chin. Mater. Med. 2005, 30, 410 (in Chinese). (张韶瑜, 孟林, 高文远, 宋乃宁, 贾伟, 段宏泉, 中国中药杂志, 2005, 30, 410.)

[2] Yang, G.-Y.; Wang, C.-X.; Huang, L.-T.; Xu, C.-L. Chin. J. Synth. Chem. 2011, 9, 337 (in Chinese). (杨国玉, 王彩霞, 黄立挺, 徐翠莲, 合成化学, 2011, 9, 337.)

[3] Fylaktakidou, K. C.; Hadjipavlou-Litina, D. J.; Litinas, K. E.; Nicolaides, D. N. Curr. Pharm. Des. 2004, 10, 3813.

[4] Putri, D. E. K.; Pranowo, H. D.; Haryadi, W. Mater. Sci. Forum 2019, $948,101$.

[5] Shi, Y.; Zhou, C.-H. Biomed. Chem. Lett. 2011, 21, 956.

[6] Atmaca, M.; Bilgin, H. M.; Obay, B. D.; Diken, H.; Kelle, M.; Kale, E. J. Physiol. Biochem. 2011, 67, 569.

[7] Zhao, H.; Neamati, N.; Pommier, Y.; Burke, T. R. J. Cheminf. 2010, $29,1002$.

[8] Weber, U. S.; Steffen, B.; Siegers, C. P. Res. Commun. Mol. Pathol. Pharmacol. 1998, 2, 193.

[9] Abdizadeh, T.; Kalani, M. R.; Abnous, K.; Tayarani, Z.; Khashyarmanesh, B. Z.; Abdizadeh, R.; Ghodsi, R.; Hadizadeh, F. Eur. J. Med. Chem. 2017, 132, 42.

[10] Yang, E. B.; Zhao, Y. N.; Zhang, K.; Mack, P. Biochem. Biophys. Res. Commun. 1999, 260, 682.

[11] Kostova, I. Curr. Med. Chem. 2005, 5, 29.
[12] Prota, A. E.; Bargsten, K.; Zurwerra, D.; Field, J. J.; Diaz, J. F.; Altmann, K. H. Science 2013, 339, 587.

[13] Romagnoli, R.; Baraldi, P. G.; Brancale, A.; Ricci, A.; Hamel, E.; Bortolozzi, R. J. Med. Chem. 2011, 54, 5144.

[14] Romeo, S. O.; Andrea, B.; Ernest, H. J. Med. Chem. 2015, 58, 3209.

[15] Anthony, N. G.; Breen, D.; Clarke, J.; Donoghue, G.; Waigh, R. D. J. Med. Chem. 2007, 50, 6116.

[16] Kumar, N.; Kumar, S.; Abbat, S.; Nikhil, K.; Pruthi, V. Med. Chem. Res. 2016, 25, 1175.

[17] Eckelbarger, J. D.; Parker, M. H.; Yap, M. C.; Buysse, A. M.; Babcock, J.M.; Hunter, R. Pest. Manage. Sci. 2016, 73, 761.

[18] Zheng, Y.-G.; Guo, J.-J.; Yu, Z.-L.; Fu, R.-K.; Huang, Y.; Wang, Y.-H.; Deng, Z.; Wu, Y. Fine Chem. Intermed. 2015, 45, 1 (in Chinese).

(郑玉国，郭晴晴，余忠林，付如凯，黄勇，王永欢，邓钊，吴用， 精细化工中间体, 2015, 45, 1.)

[19] Wen, J.-W.; Li, Y.; Su, Z.-Y.; Wan, L. Chemistry 2019, 82, 350 (in Chinese). (温借雯，黎勇，苏正颖，万丽，化学通报, 2019, 82, 350.)

[20] Zhang, M.-Q.; Liu, B.-K.; Lei, Q.; Long, Y. Chin. J. Org. Chem. 2019, 39, 1064 (in Chinese). (张明千, 刘斌凯, 雷强, 龙跃, 有机化学, 2019, 39, 1064.)

[21] Fu, D.-J.; Song J.; Hou Y.-H.; Zhao R.-H.; Li J.-H.; Mao R.-W.; Zhang Y.-B.; Liu H.-M. Eur. J. Med. Chem. 2017, $138,1076$.

[22] Fu, D.-J.; Yang, J.-J.; Li, P.; Hou, Y.-H.; Huang, S.-N, Pham, V.; Song, L. K.; Zi, X. L.; Xue, W.-L. Eur. J. Med. Chem. 2018, 157, 50 . 